\title{
Two-Photon Emissive Dyes Based on Push-Pull Purines Derivatives: Toward the Development of New Photoluminescence Bioprobes
}

Leandro H. Zucolotto Cocca ${ }^{\mathrm{a}}$, Luis M. G. Abegão ${ }^{\mathrm{b}}$, Lucas F. Sciuti ${ }^{\mathrm{a}}$, Roxane Vabre ${ }^{\mathrm{c}, \mathrm{d}}$, Jonathas de Paula Siqueira $^{\mathrm{e}}$, Kenji Kamada ${ }^{\mathrm{f}}$, Cleber R. Mendonca ${ }^{\mathrm{a}}$, Sandrine Piguel ${ }^{\mathrm{c}, \mathrm{d}}$ and Leonardo De Boni ${ }^{\text {a,* }}$

a Instituto de Física de São Carlos, Universidade de São Paulo, CP 369, 13560-970 São Carlos, SP, Brazil

b Department of Radiology \& Biomedical Imaging, School of Medicine, Yale University, New Haven, Connecticut 06520, USA

c Université Paris-Saclay, CNRS, Inserm, 91405, Orsay, France

d Institut Curie, CNRS, Inserm, 91401, Orsay, France

e Universidade Estadual de Campinas, Instituto de Física "Gleb Wataghin", Cidade Universitária Zeferino Vaz Barão Geraldo, 13083-859 Campinas, SP, Brazil

f Nanomaterials Research Institute, National Institute of Advanced Industrial Science and Technology (AIST), Ikeda, Osaka 563-8577, Japan

* Author to whom correspondence should be addressed: deboni@ifsc.usp.br 


\section{Supporting information}

\section{Experimental section}

\section{a) Molar absorptivity and fluorescence emission spectrum}

In order to determine the photophysical properties related to linear optical spectroscopy, the nine Push-pull 6-amino-8-styryl purines were dissolved at a concentration about $10^{-6} \mathrm{~mol} / \mathrm{L}$ in dichloromethane (DCM) (3a, 3b, 3c, 3d, 3e, 3f, 3g) and dimethyl sulfoxide (DMSO) (7a and 7b). A Shimadzu UV-Vis 1800 spectrophotometer was employed to measure the absorption spectrum, more specifically, the molar absorptivity spectrum $(\varepsilon=A(\lambda) / l C$, where $A(\lambda)$ is the absorbance spectrum, $l$ the cuvette optical path and $C$ is the concentration in mol/l). A F-7000 Hitachi fluorescence spectrophotometer was used to collect the fluorescence emission spectrum. The samples were placed in a $2 \mathrm{~mm}$ quartz cuvette for absorption spectrum measurements and in a $1 \mathrm{~cm}$ quartz cuvette for fluorescence spectrum determination. Molar absorption are presented in Table S1.

\begin{tabular}{llllllllll}
\multicolumn{2}{c}{$3 a$} & $3 b$ & $3 c$ & $3 d$ & $3 e$ & $3 f$ & $3 g$ & $7 a$ & $7 b$ \\
\hline$\varepsilon\left(10^{4} \mathrm{Lcm}^{-1}\right.$ & $2.1,270$ & $2.0,264$ & $2.3,276$ & $2.4,270$ & $1.45,269$ & $1.75,263$ & $2.8,273$ & $3.0,270$ & $1.5,269$ \\
$\left.\mathrm{~mol}^{-1}\right), \quad \lambda_{\text {abs }}$ & $1.3,297$ & $1.2,286$ & $1.5,303$ & $1.2,298$ & $1.2,294$ & $1.1,291$ & $1.75,304$ & $2.0,291$ & $0.8,292$ \\
$(\mathrm{~nm})$ & $1.9,370$ & $1.5,357$ & $1.9,391$ & $1.6,375$ & $1.55,370$ & $1.6,357$ & $2.4,383$ & $1.9,373$ & $1.6,367$ \\
\hline$\lambda_{\text {em }}(\mathrm{nm})$ & 464 & 451 & 489 & 467 & 466 & 451 & 486 & 493 & 479
\end{tabular}

Table S1: Molar absorption $\left(\times 10^{4} \mathrm{~L} \mathrm{~cm}^{-1} \mathrm{~mol}^{-1}\right)$ and the respective wavelength (nm) and the wavelength (nm) at the maximum of normalized fluorescence emission.

Figure S1 show molar absorptivities (black lines), fluorescence emission spectra (red lines) and also for each the fluorescence quantum yield $\left(\phi_{f}\right)$ values.
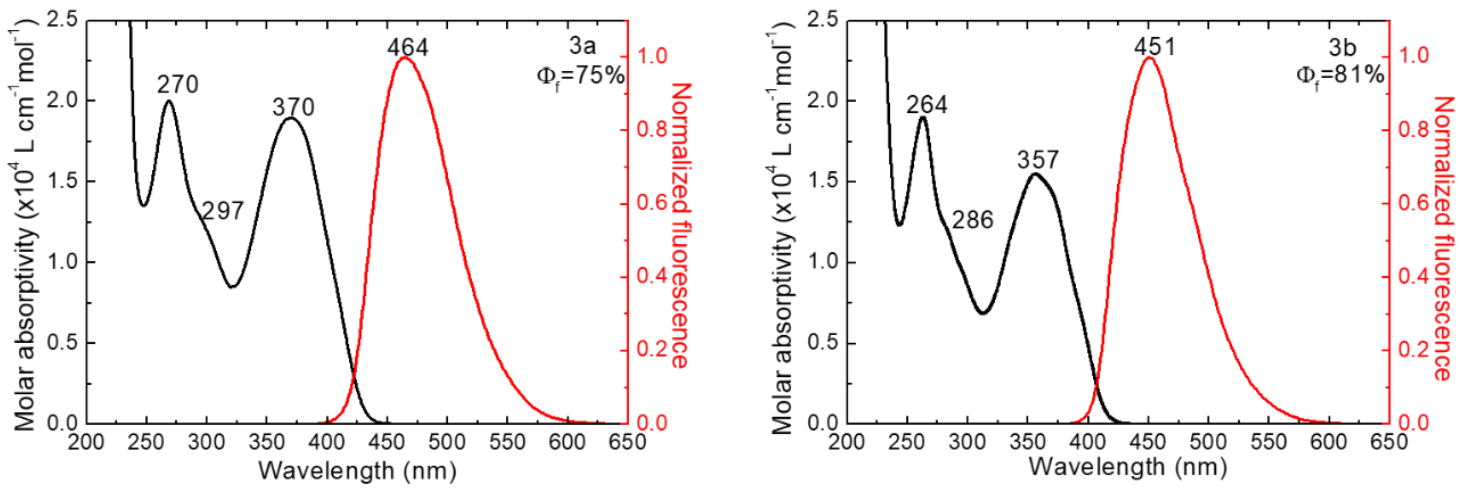

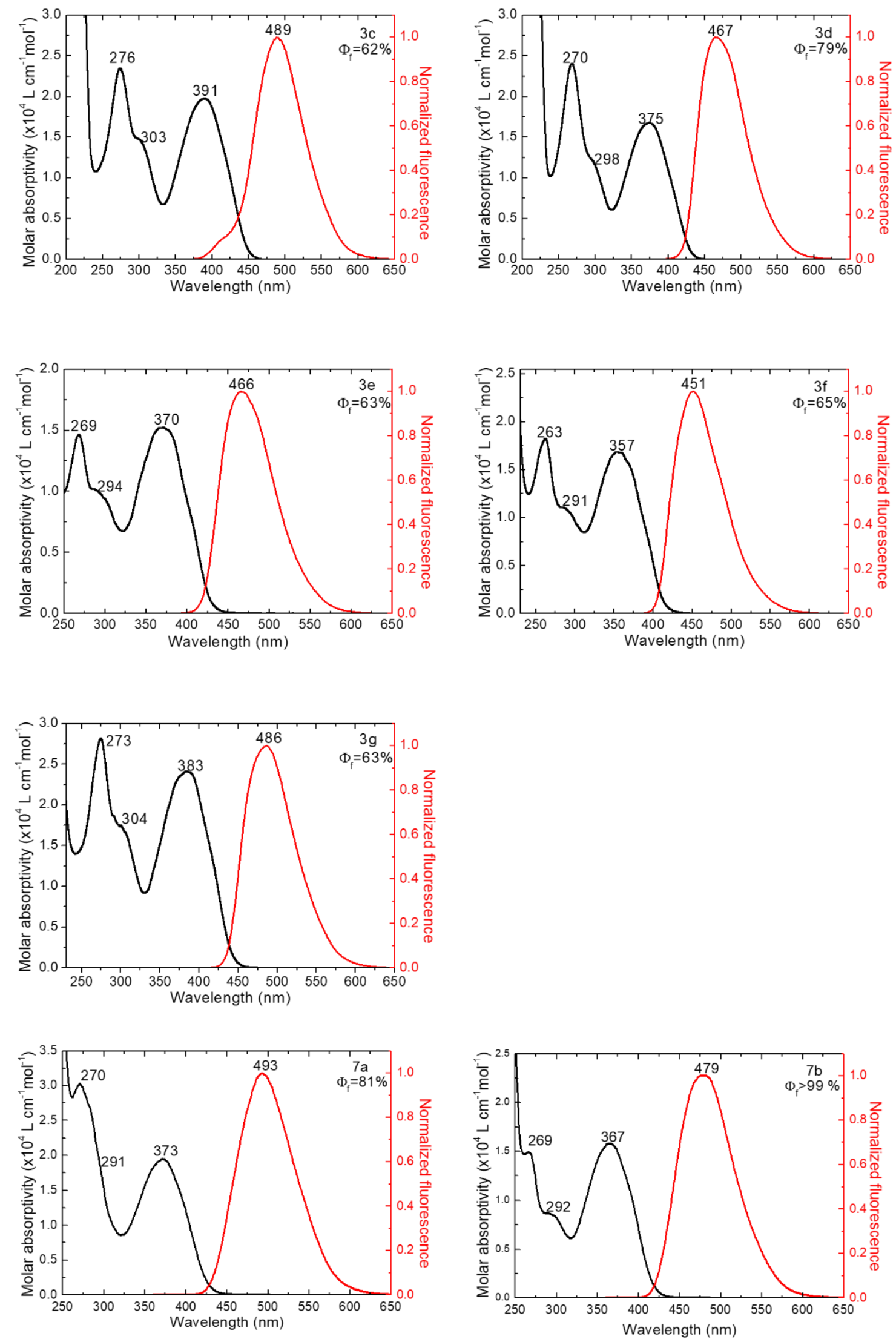

Figure S1: Molar absorptivities (black lines) and fluorescence emission spectra (red lines). Fluorescence quantum yield $\left(\phi_{f}\right)$ values are presented as well. 


\section{b) Fluorescence lifetimes}

Fluorescence lifetimes $\left(\tau_{f}\right)$ were determined by employing time-resolved fluorescence technique ${ }^{1-5}$, for that, a Ti-Sapphire Clark MXR-2001 laser generating pulses of $150 \mathrm{fs}$ at $775 \mathrm{~nm}$ and $1 \mathrm{kHz}$ repetition rate was used and doubled in frequency in a BBO crystal. For these measurements, the samples were also placed in the $2 \mathrm{~mm}$ quartz cuvette. The emission signal was collected perpendicularly to the excitation beam by an optical fiber, registered by a silicon photodetector of $0.7 \mathrm{~ns}$ of minimum temporal resolution. The fluorescence signal decay was acquired by a digital oscilloscope. Through the acquired signal, it is possible to determine the $\tau_{f}$ by fitting a monoexponential curve $\mathrm{I}(\mathrm{t})=\mathrm{I}_{0} \mathrm{e}^{-\mathrm{t} / \tau_{\mathrm{f}}}$, in which $I(t)$ is the intensity of the fluorescence signal and $I_{0}$ is the intensity at $t=0$ which represents the time at maximum fluorescence intensity. Figure SI2 shows the fluorescence decays (black circles) and mono-exponential fitting (red lines) to the nine purines samples. Also, it is possible to see the fluorescence lifetime values. During the measurements sets, photodegradation of the samples were not observed, it was checked by measuring the absorption before and after each measurements.
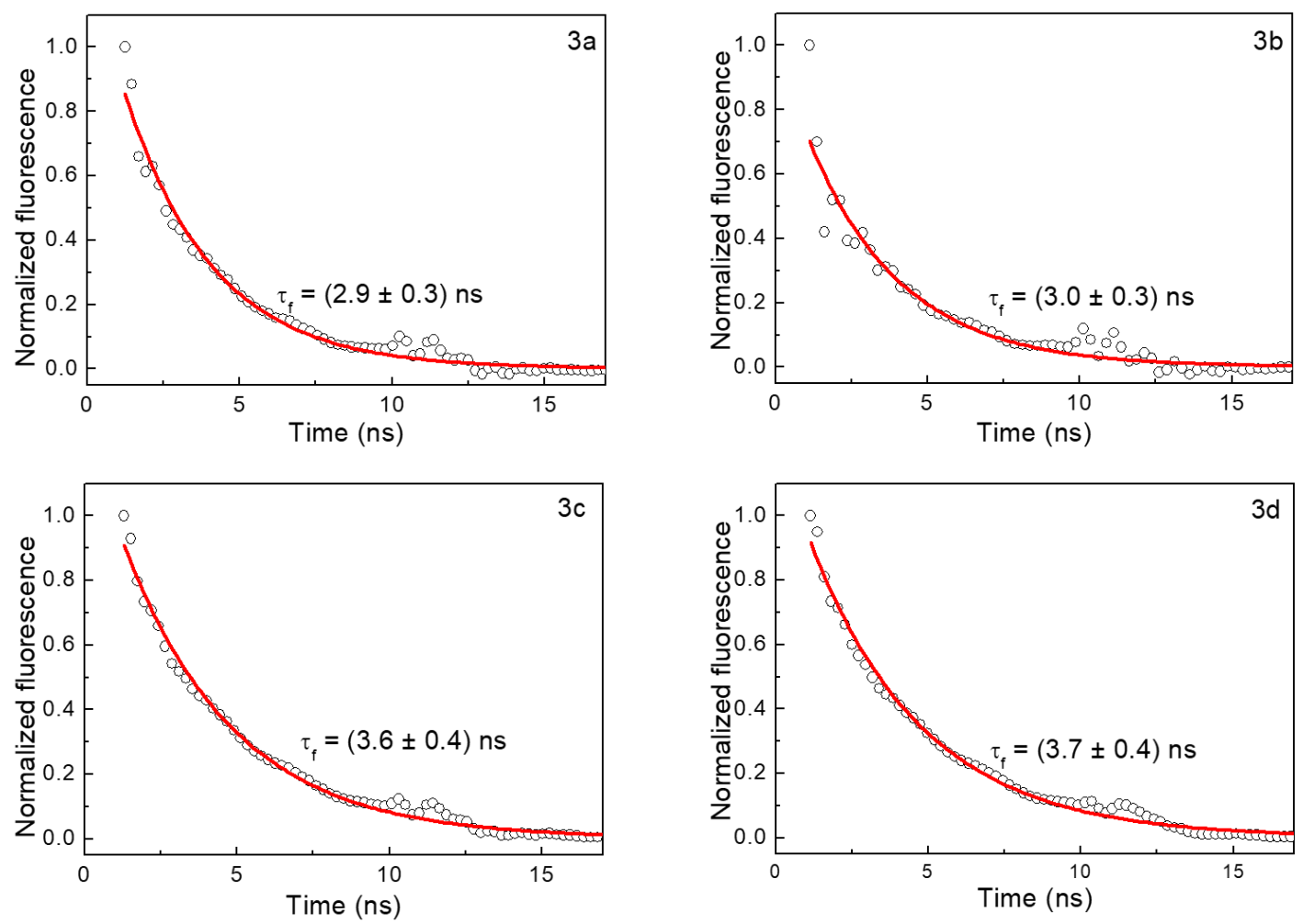

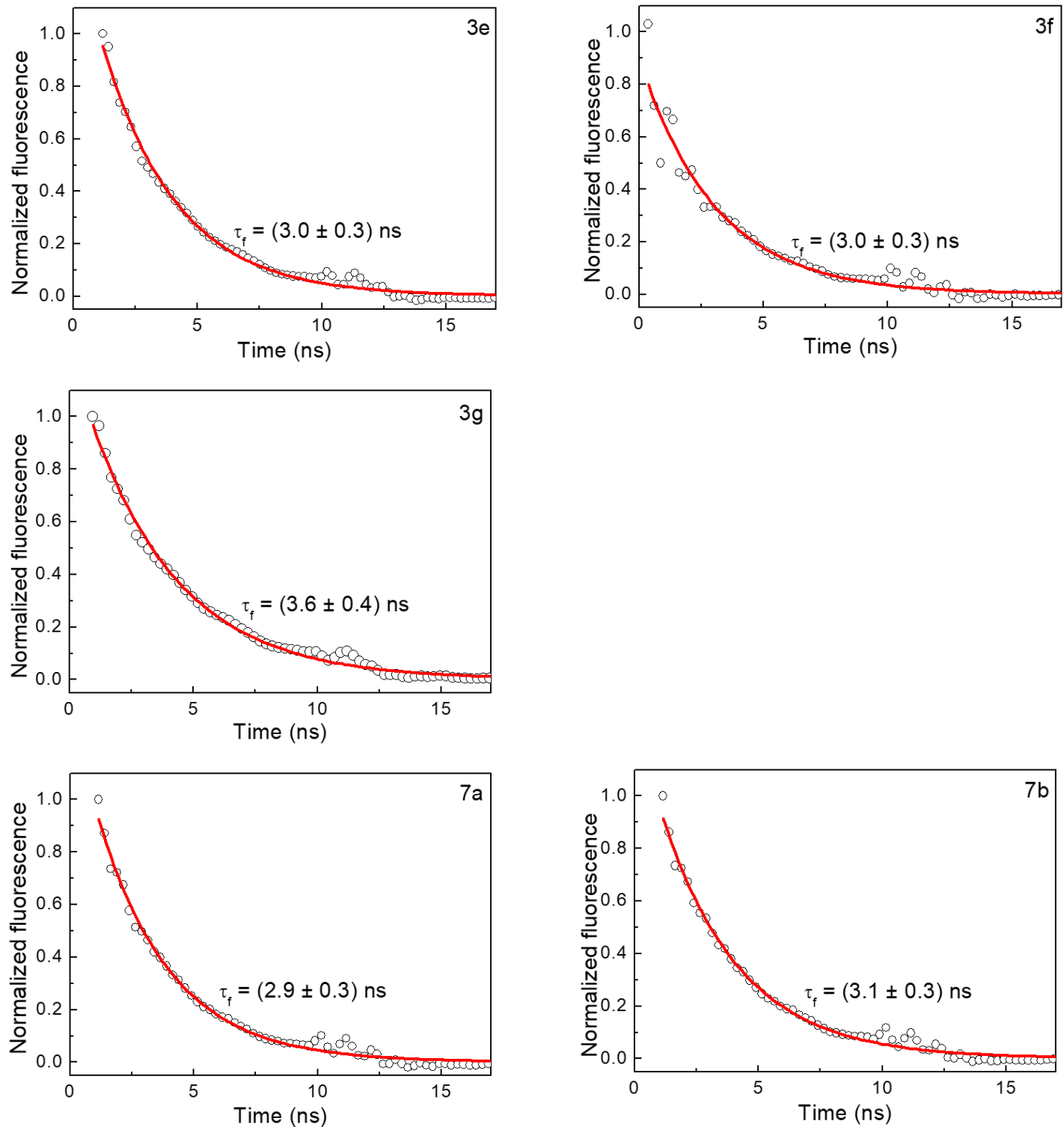

Figure S2: Fluorescence decays (black circles) and the monoexponential fittings (red lines) for the fluorescence lifetimes determination.

The fluorescence lifetime is defined by the sum of the radiative and nonradiative decay rates from the first excited estate to the ground state $\left(k_{r}=\frac{1}{\tau_{r}}\right.$ and $k_{n r}=\frac{1}{\tau_{n r}}$, respectively, in which, $\tau_{n r}$ is the nonradiative decay time). In this way, it is possible to write that $\frac{1}{\tau_{f}}=\frac{1}{\tau_{r}}+\frac{1}{\tau_{n r}}$, and consequently, it is possible to define $\tau_{r}=\frac{\tau_{f}}{\phi_{f}} \cdot k_{r}$ and $k_{n r}$ values show the preferential decay path to which the population from the first excited state decays to the ground state. In other words, $k_{r}$ and $k_{n r}$ show if the decay of the first excited state to the ground state is more likely to occur via radiative (by photon emission) or via nonradiative (by vibrational relaxation). 


\section{c) Solvatochromism measurements}

To determine the difference between excitedand ground states permanent electric dipole moment $\left(\Delta \mu_{01}=\mu_{11}-\mu_{00}\right)$, solvatochromic measurements ${ }^{3,6}$ were performed. For $\Delta \mu_{01}$ determination, it was used the Lippert-Mataga equation: $\Delta v=$ $\frac{\Delta \mu_{01}{ }^{2}}{c h a^{3}} \Delta F^{3,6} . \Delta v$ is the difference between absorption and emission peaks (in $\mathrm{cm}^{-1}$ ), $c$ is the speed of light, $h$ the Planck's constant, $a^{3}$ is the cubic radius the molecule possesses when surrounded by the solvent and $\Delta F$ is the Onsager polarity function, given by $F=$ $2\left[\frac{\varepsilon-1}{2 \varepsilon+2}-\frac{n^{2}-1}{2 n^{2}+2}\right]$, in which $n$ is the solvent refractive index and $\varepsilon$ is the solvent dieletric constant. For the $a^{3}$ determination, it was used the Smoluchowski-Einstein equation ${ }^{3,7}$ which relates the hydrodynamic volume of the molecular structure to the fluorescence anisotropy ( $\mathrm{r}$ given by $\operatorname{Vol}_{h}=\frac{\tau_{f} K T}{\eta\left({ }^{0.4} / r^{-1}\right)}=\frac{4}{3} \pi a^{3}$, where $\tau_{f}$ is the fluorescence lifetime, $K$ is the Boltzmann constant, $T$ is the room temperature in $\mathrm{K}, \eta$ is the solvent viscosity (in Poise), $r$ is the fluorescence anisotropy and the value of 0.4 is due to the maximum value that the anisotropy of a such material can reach, which occurs in the case that the excitation and emission dipoles moments are parallel. Lastly, to determine $\Delta \mu_{01}$, it was necessary to calculate the fluorescence anisotropy ${ }^{3,6}$ defined by $r=\frac{I_{v v}-G I_{v h}}{I_{v v}+2 G I_{v h}}$, in which $G=\frac{I_{h v 3}}{I_{h h}}$. Here, $I$ is the emission intensity and $G$ is the sensitivity of the excitation and emission channels of the fluorescence spectrophotometer. The indexes $v v, v h, h v$ and $h h$ refers to the polarizations ( $v=$ vertical and $h=$ horizontal) in each of these excitation and emission channels. Fluorescence anisotropy (black circles) are depicted in Figure SI3, the black lines, at the same figure, represents the absorption spectrum.
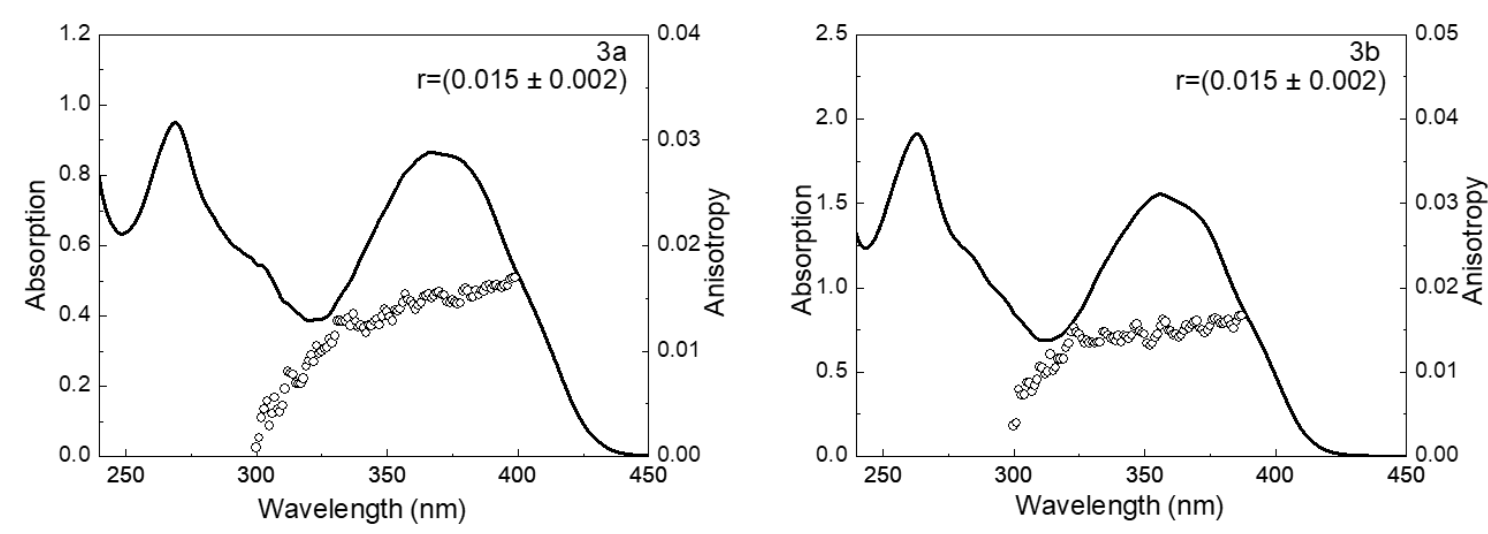

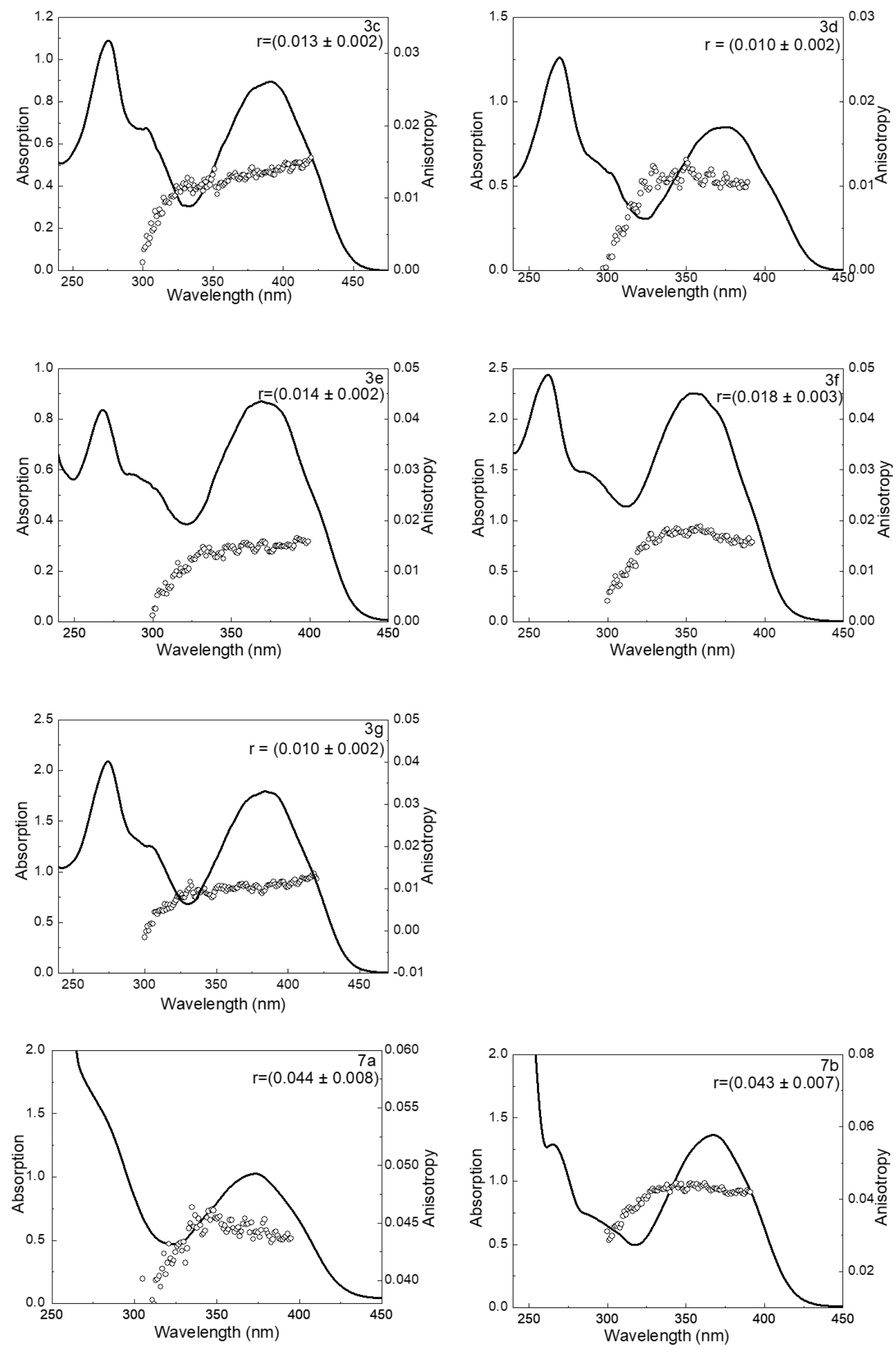

Figure S3: Absorption spectrum (black line) and fluorescence anisotropy (black circles) for nine purine samples dissolved in DCM (3a-3g) and DMSO (7a and 7b). 
In the Figure SI4, it is possible to note the solvatochromic measurements, or in other words, it is possible to see the shifts of normalized absorption and the normalized fluorescence as a function of different solvents.
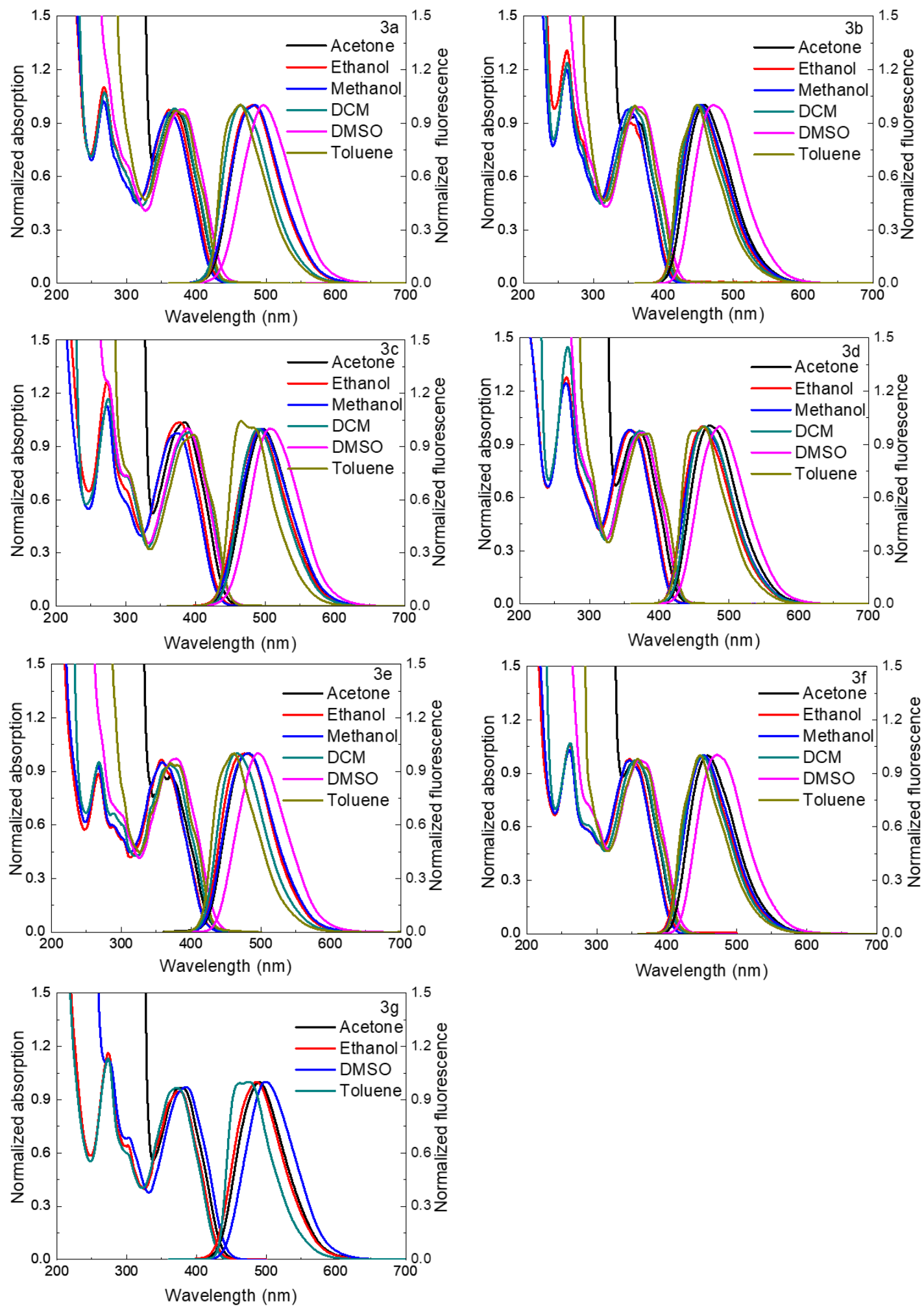

Figure S4: Solvatochromics measurements of 3a-3g samples for distinct solvents, the absorption band is located at 320 up to $450 \mathrm{~nm}$ region, and the fluorescence emission between 450 up to $600 \mathrm{~nm}$. Solvatochromics measurements of $7 \mathrm{a}$ and $7 \mathrm{~b}$ molecules were performed by Vabre et al ${ }^{8}$. 
Finally, Figure SI5 shows the linear fittings (red lines) used to determine $\frac{\Delta v}{\Delta F}$ for the nine purines molecules studied in this work.
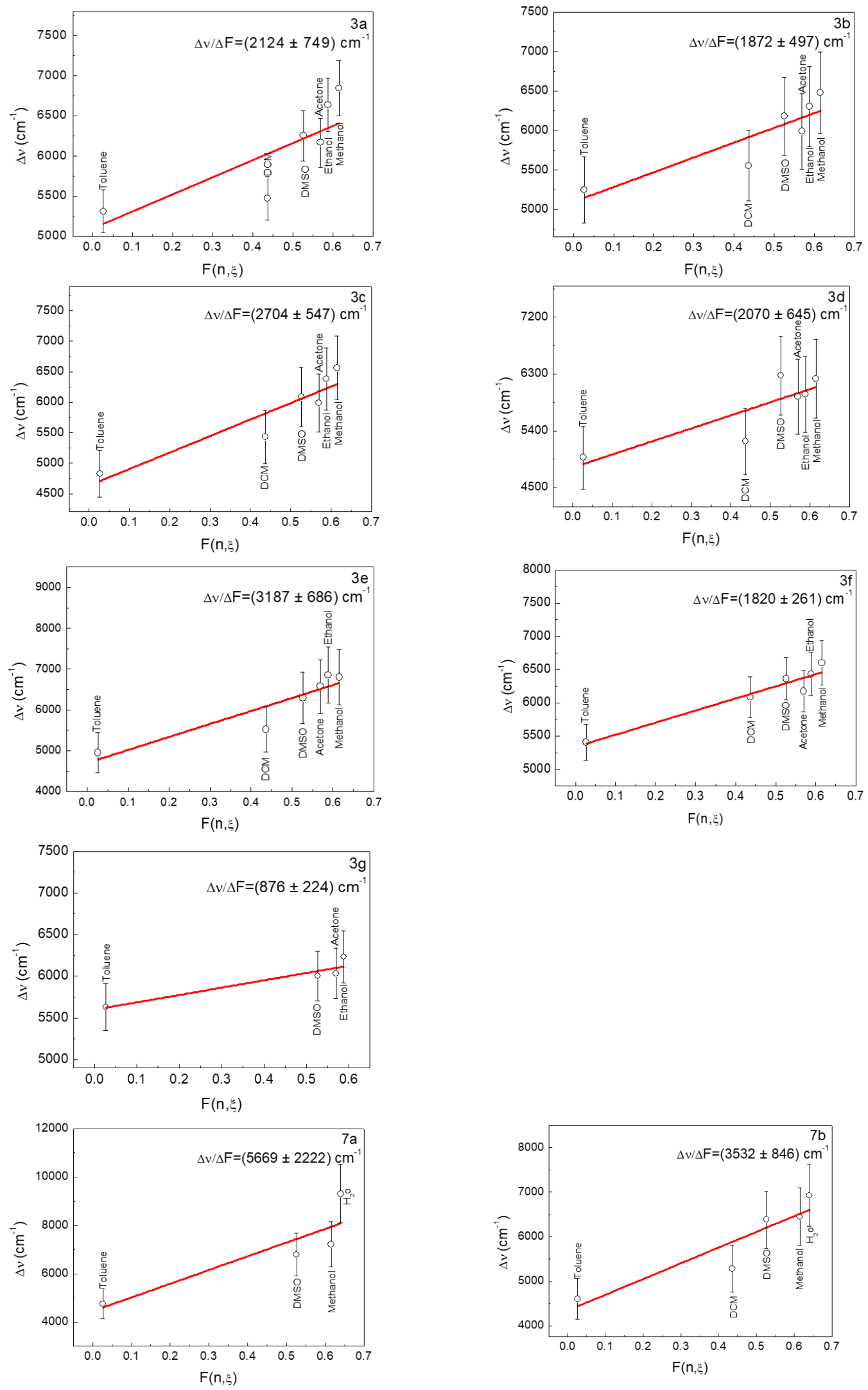

Figure S5: Linear fittings use to determine $\frac{\Delta v}{\Delta F}$, values presented in each graph. 
$\mu_{00}, \Delta \mu_{02}$ and $\Delta \mu_{03}$ values were determined also by solvatochromic measurements employing the follow equation: $\Delta \vec{\mu}_{f g} \cdot \vec{\mu}_{f g}=-\frac{3}{4 \pi} h c \frac{\partial v_{A i}}{\partial F} V_{o l}{ }^{9} \quad(i=1,2$ and 3). In this equation, $\frac{\partial v_{A i}}{\partial F}$ refers to the energy variation of absorption band peaks as a function of the Onsager polarity function $(\Delta \mathrm{F})$, the index $i=1$ refers to the first band (lower energy band), the index $i=2$ refers to the second band and $i=3$ refers to the third band (higher energy band), $\mathrm{Vol}$ is the hydrodynamic volume of the molecular structure previously determined by Smoluchowski-Einstein equation, $h$ is the Planck constant and $c$ is the speed of light. Firstly, $\mu_{00}$ was determined by using the values of $\Delta \vec{\mu}_{01}$ and the $\frac{\partial v_{A 1}}{\partial F}$ (referent to the lower energy band peaks, $i=1$ ). It is important to highlight that the values of $\Delta \vec{\mu}_{01}$ were previously determined through Lippert-Mataga equation. Once $\mu_{00}$ was obtained, we quantified $\Delta \mu_{02}$ value with the same equation, considering the second absorption band in which $\frac{\partial v_{A 2}}{\partial F}(i=2)$ is evaluated. For $\Delta \mu_{03}$ the same procedure was used considering the third absorption band, $\frac{\partial v_{A 3}}{\partial F}(i=3)$. The values of $\mu_{00}, \Delta \mu_{02}$ and $\Delta \mu_{03}$ are present in the Table $\mathrm{S} 2$. The errors associated with this values are considered high (about 30\%) due to difficulty to determine the absorption bands peaks position. Consequently, this values were used to guide the SOS model in a way to give the range of the magnitude allowed to adjust the 2PA spectra.

$\begin{array}{llllllll} & \mathbf{3 a} & \mathbf{3 b} & \mathbf{3 c} & \mathbf{3 d} & \mathbf{3 e} & \mathbf{3 f} & \mathbf{3 g} \\ \boldsymbol{\mu}_{\mathbf{0 0}} \text { (D) } & 2.5 & 2.5 & 2.9 & 2.7 & 2.3 & 2.7 & 2.7 \\ \boldsymbol{\Delta} \boldsymbol{\mu}_{\mathbf{0 2}} \text { (D) } & 4.2 & 4.9 & 3.2 & 3.2 & 4.7 & 3.9 & 2.1 \\ \boldsymbol{\Delta} \boldsymbol{\mu}_{\mathbf{0 3}} \text { (D) } & 4.0 & 4.2 & 4.9 & 5.9 & 4.2 & 5.7 & 2.3\end{array}$

Table S2: $\mu_{00}, \Delta \mu_{02}, \Delta \mu_{03}$, determined through solvatochromic measurements. The error associated are around $30 \%$. D represents Debye units.

\section{d) Transition electric dipole moment}

The transition electric dipole moments between ground and first excited state $\left(\mu_{01}\right)$, ground and second excited state $\left(\mu_{02}\right)$ and ground and third excited state $\left(\mu_{03}\right)$ were determined through linear absorption spectrum previously determined. $\mu_{0 i}$ value is given by the following equation $\mu_{0 i}=\sqrt{\frac{3}{8 \pi} \frac{h c}{N} \frac{n}{L^{2}} \frac{1}{v_{0 i}} \int \alpha_{0}(v) d v^{3}}, 10$ in which $\mathrm{L}=\frac{3 n^{2}}{2 n^{2}+1}$ is the Onsager local field factor, $n$ is the solvent refractive index (1.4244 for DCM and 1.479 for DMSO), $h$ is the Planck's constant, $c$ is the speed of light in vacuum, $N$ is the solution concentration (in molecules $/ \mathrm{cm}^{3}$ ), $v_{0 i}$ is the transitions frequencies in $\mathrm{Hz}$ 
related to transitions from the ground state to the first, second and third excited state ( $i=1,2$ and 3 respectively), and finally, $\alpha_{0}(v)$ is the absorption coefficient (in $\mathrm{cm}^{-1}$ ) determined from the linear absorption spectrum $\left(\alpha_{0}(v)=A(\lambda) \ln (10) / L\right)$, in which $A(\lambda)$ is the absorbance at wavelength $\lambda$ and $l$ is the cuvette path length, also it was deconvoluted into Lorentizian lines to obtain the $\int \alpha_{0}(v) d v$, the $\alpha_{0}(v)$ is the amplitude factor. Figure S6 shows the Lorentizian decovolutions (green lines) on the absorption coefficient spectra, $\alpha_{0}$ (black line), the red line represents the fitting between Lorentizian deconvolution and the experimental $\alpha_{0}$. In each graph of Fig S6, it is possible to see the values of $\mu_{01}, \mu_{02}$ and $\mu_{03}$.
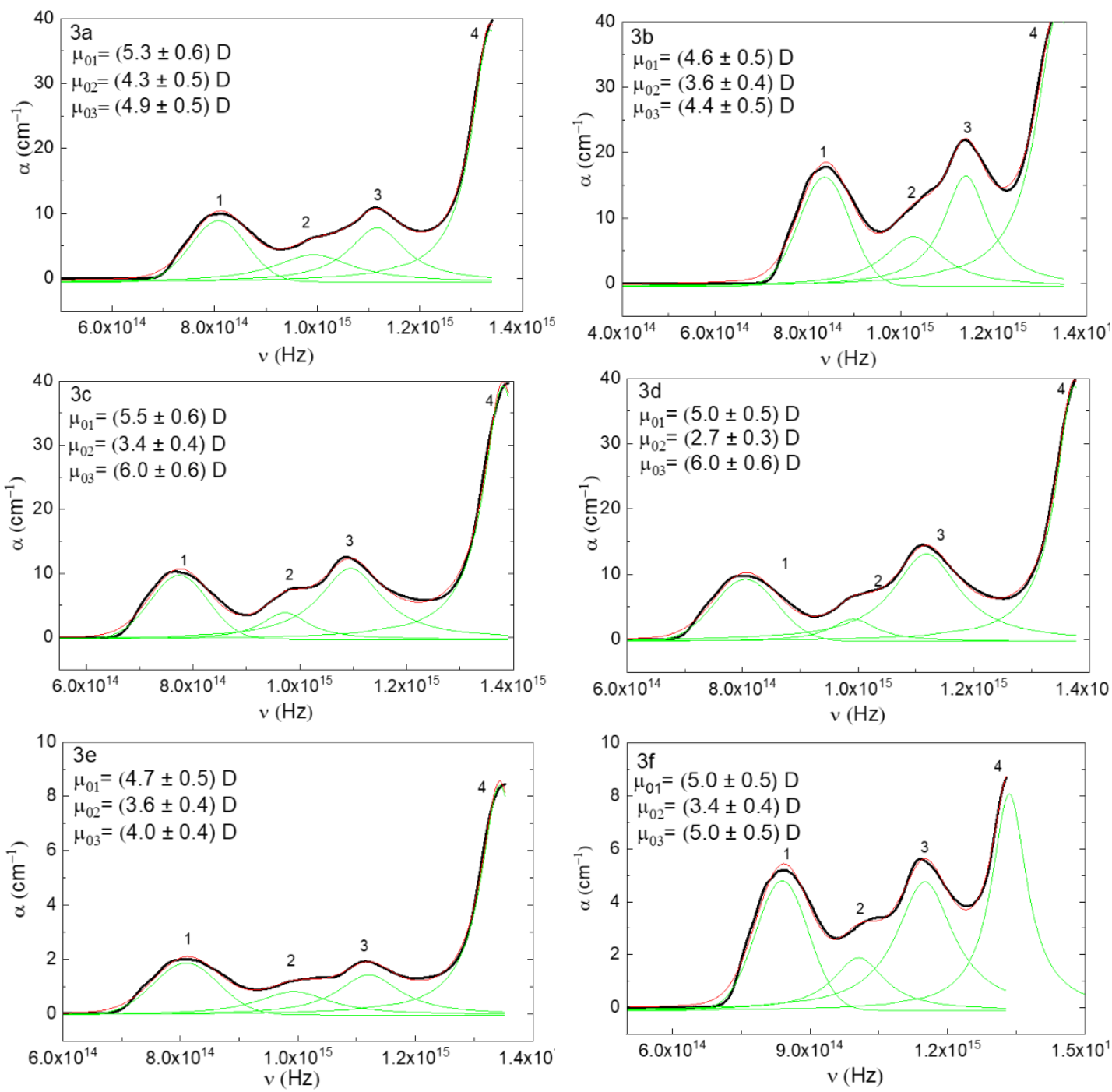

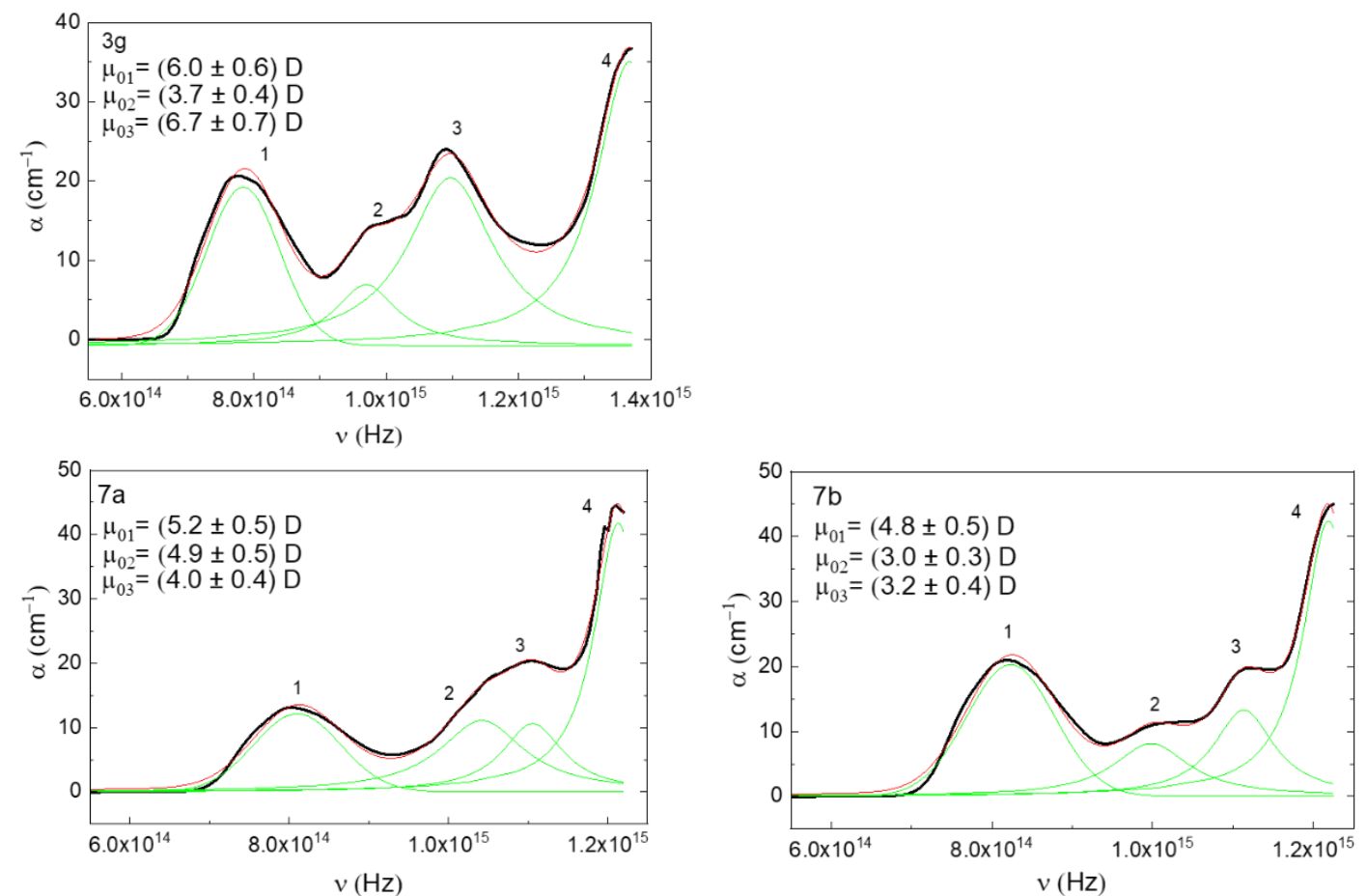

Figure S6: Lorentzians deconvolutions (green lines) and fitting adjustment (red line) on the absorption coefficient $\left(\alpha_{0}(v)\right)$ spectrum (black line), also each graph shows and calculated transition dipole moments.

\section{e) Two-photon absorption (2PA) cross-section}

Once completed the experiments related to linear spectroscopy, it was performed experiments related to the nonlinear optical ones. In this case, it was determined the two-photon absorption (2PA) cross-section spectrum $\left(\sigma_{2 P A}(\lambda)\right)$ through the Z-scan technique ${ }^{10-12}$. This technique is based on the normalized transmittance (NT) acquisition while the sample is translated around the focal region. The NT is the sample transmittance near focus divided by its transmittance far from focus. When the sample is far from focus, only linear effects are observed due to the low intensity of the laser beam and, when the sample is near the focus, nonlinear effects become more pronounced. NT is acquired by a silicon photodetector and a homemade Labview program captures the data and controls the experiment. The 2PA cross-section can be determined through the equation: $T(z)=\frac{1}{\sqrt{q} q_{0}(z, 0)} \int_{-\infty}^{\infty} \ln \left[1+q_{0}(z, 0) e^{-\tau^{2}}\right] d \tau, q_{0}=$ $\beta I_{0} L\left(1+\left(\frac{z^{2}}{z_{0}^{2}}\right)\right)^{-1}$ and $\sigma_{2 P A}=\frac{\hbar \omega \beta}{N} \cdot \beta$ is the nonlinear absorptioncoefficient, $z_{0}$ is the beam Rayleigh length at the focal region, $z$ is the sample position, $I_{0}$ is the laser intensity at the focus, and $L$ is the cuvette path length, in our case a $2 \mathrm{~mm}$ cuvette. The 2PA cross-section spectrum was determined via $775 \mathrm{~nm}$ and 150 fs pulses generated by a Clark MXR-2001 Ti-Sapphire laser working at $1 \mathrm{kHz}$ which pumps an optical 
parametric amplifier (TOPAS Quantronix) delivering radiation from $460 \mathrm{~nm}$ up to 2600 nm. Wavelengths were chosen depends on the two-photon absorption region to be studied. For the present samples, the $\sigma_{2 P A}$ spectrum was measured for all nine samples ranging from $460 \mathrm{~nm}$ up to $900 \mathrm{~nm}$ in steps of $10 \mathrm{~nm}$.

In our Z-scan setup, a system of three dichroic mirrors is used to filter the desired wavelength from spurious wavelengths generated on TOPAS. Besides that, it is important to say that each wavelength used to measure the $2 \mathrm{PA}$ spectrum are spatial filteredby a $60 \mu \mathrm{m}$ pinhole in order to obtain a Gaussian spatial profile. Before the Zscan setup, a thin $4 \%$ reflection beam spliter is used to direct a portion of laser to a reference silicon photodetector $\left(1 \mathrm{~cm}^{2}\right.$ of area), which is used to measure power fluctuation of the laser during the measurementand normalize each point the Z-Scan signal by the correlated fluctuation. After this stage, the laser beam reaches two thin gridewire polarizers (the first one is used to control the average power of the laser on the sample, and the second one is used to fixlight polarization to be always the same. After this point, a $15 \mathrm{~cm}$ convergent lens is used to focus the laser beam in the sample, which is translated through the focal region. After it, a short convergent lens is used to focus all the transmitted light trhough the sample in a second silicon photodetector (1 $\mathrm{cm}^{2}$ of area), as known as Open aperture Z-scan configuration. The signal of both detectors are averaged in two individual Lock-in amplifiers. The signal is acquire by a homemade Labview program, witch also control the entire experiment. Each point of the Z-scan signature is an average of 1000 laser pulses (1 second). The 2PA cross sections spectrum for each sample was measured five times at different days. Distinct laser average power were used for each wavelength ranged between 0.04 up to $0.15 \mathrm{~mW}$ $(0.04 \mu \mathrm{J}$ up to $0.15 \mu \mathrm{J})$. From time to time, our Z-Scan setup is calibrated by using 1 $\mathrm{mm}$ thikness of fused silica and also Rhodamine $6 \mathrm{G}$ or B.

Figure SI7 shows (symbols) the experimental NT for four distinct wavelengths for molecule $3 \mathrm{a}, 3 \mathrm{c}, 3 \mathrm{~d}$ and $3 \mathrm{f}$, as well as the adjustment (solid lines) used to obtain 2PA cross section $\left(\sigma_{2 P A}(\lambda)\right)$. All the other samples presented the similar NT profile and dispersion. It is important to mantion that during the measurements sets, photodegradation of the samples were not observed, it was checked by measuring the absorption before and after each measurements for all samples. 

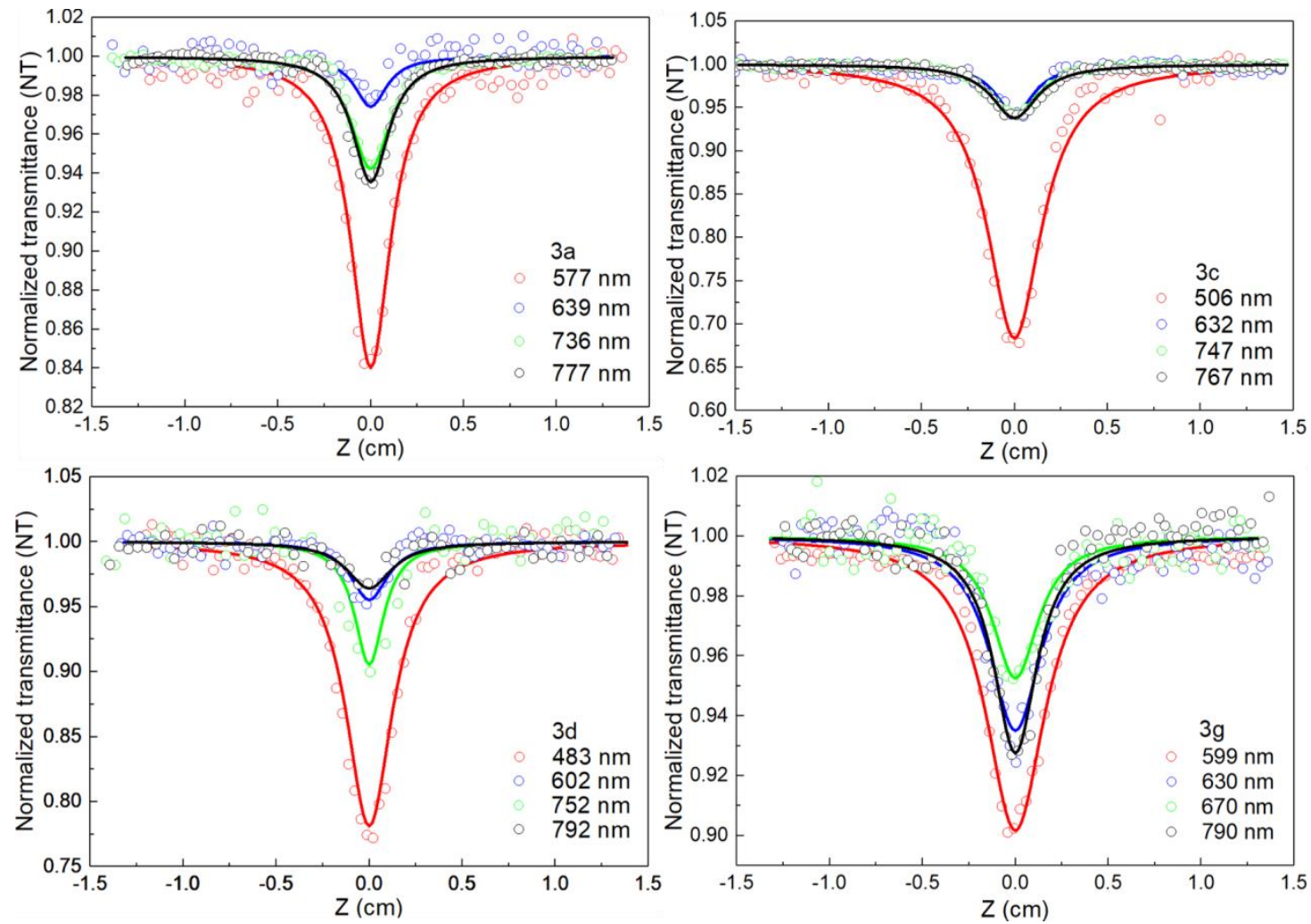

Figure S7: Normalized Transmittance (symbols) as a function of $\mathrm{Z}(\mathrm{cm})$ for molecule $3 \mathrm{a}, 3 \mathrm{c}, 3 \mathrm{~d}$ and $3 \mathrm{~g}$ for distinct wavelengths. Solid lines represent the fittings by using the equation described in this section.

\section{f) Transient absorption (Pump and Probe technique)}

Pump and probe technique was used to determine the transient absorption of nine purines samples, this technique is able to discriminate the population dynamics of excited states as well the lifetimes of excited states through transient absorption monitoring. Here, it was used as pump beam the second harmonic of $775 \mathrm{~nm}$ (from laser Clark MXR) generated in a BBO thin crystal (about 390nm and $120 \mathrm{fs}$ ), and a white light continuous as probe beam $(470 \sim 700 \mathrm{~nm}$, generated by the incidence of the $1 \%$ of the $750 \mathrm{~nm}$ laser beam in a sapphire crystal), in this way, it was possible investigate the presence of absorptions of excited states between 470 700 $\mathrm{nm}$. The pump beam induces an excitation of population and the probe beam (unable to excite population due to low intensity) monitors the decay of this population for a spectral range $(470 \sim 700 \mathrm{~nm})$, besides that, the probe beam is delayed in time of pump beam by a translation mechanism and it is possible to discriminate the changes in absorption temporally. Therefore, the variation of the lag time related with pump and the probe beam may discriminate the temporal evolution of nonlinear processes. In this work, it was monitored the normalized transmittance $(\mathrm{TN})$ of samples as a function of wavelength 
and delay time between pump and probe beams. The TN is the transmittance measure with pump off divided by transmittance with the pump on. Consequently, if TN presents an intensity variation as a function of wavelength, this may mean the presence of absorption of excited states. Figure S8 shows the TN variation as a function of wavelength and time. It is possible to see that $\mathrm{TN}$ presents a variation in a spectral range between 540-640 $\mathrm{nm}$, discriminating the presence of an absorption of excited states between this spectral range. During the measurements sets, photodegradation of the samples were not observed, it was checked by measuring the absorption before and after each measurements.
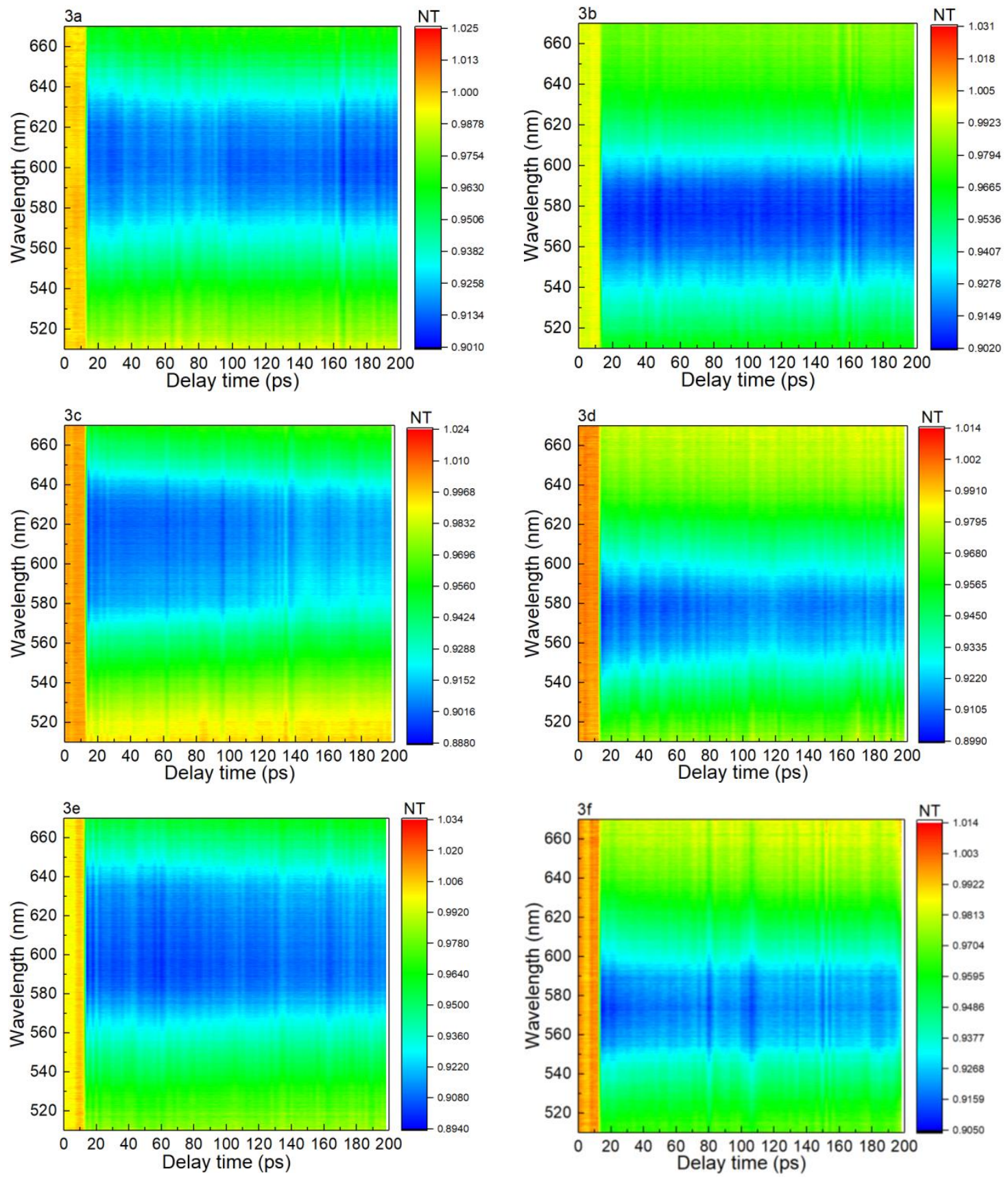

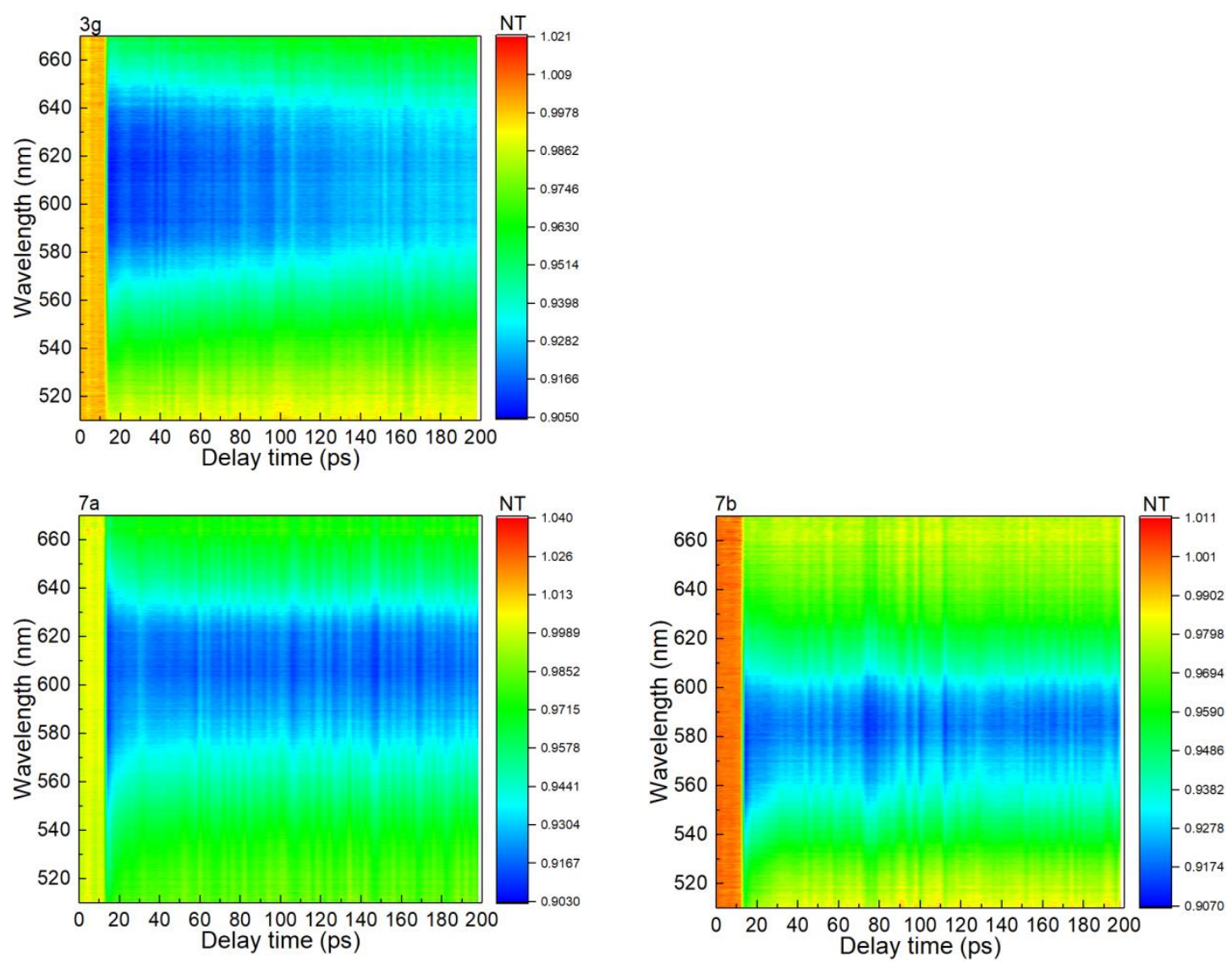

Figure S8: TN as a function of wavelength and time determined by pump and probe technique, the blue color means the largest variation of $\mathrm{TN}$ representing an absorption of excited state in this spectral region.

\section{Computation details}

a) Simulation of the one- and two-photon absorption spectra in solvent and gas-

\section{phase media}
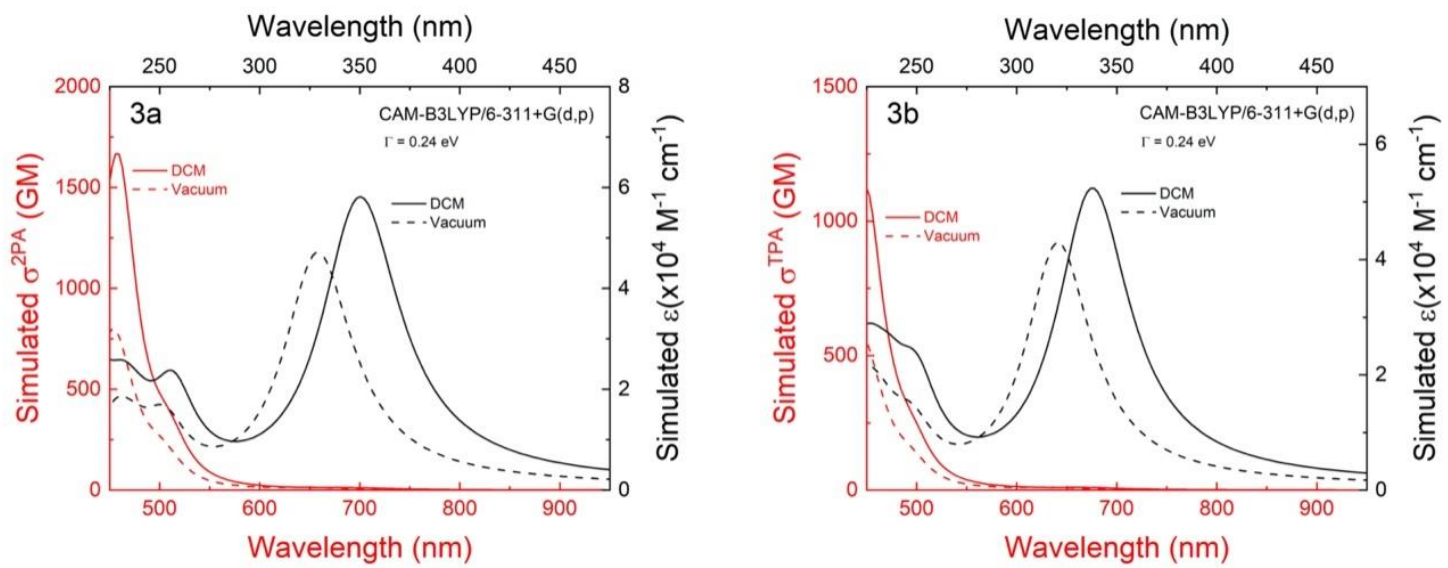

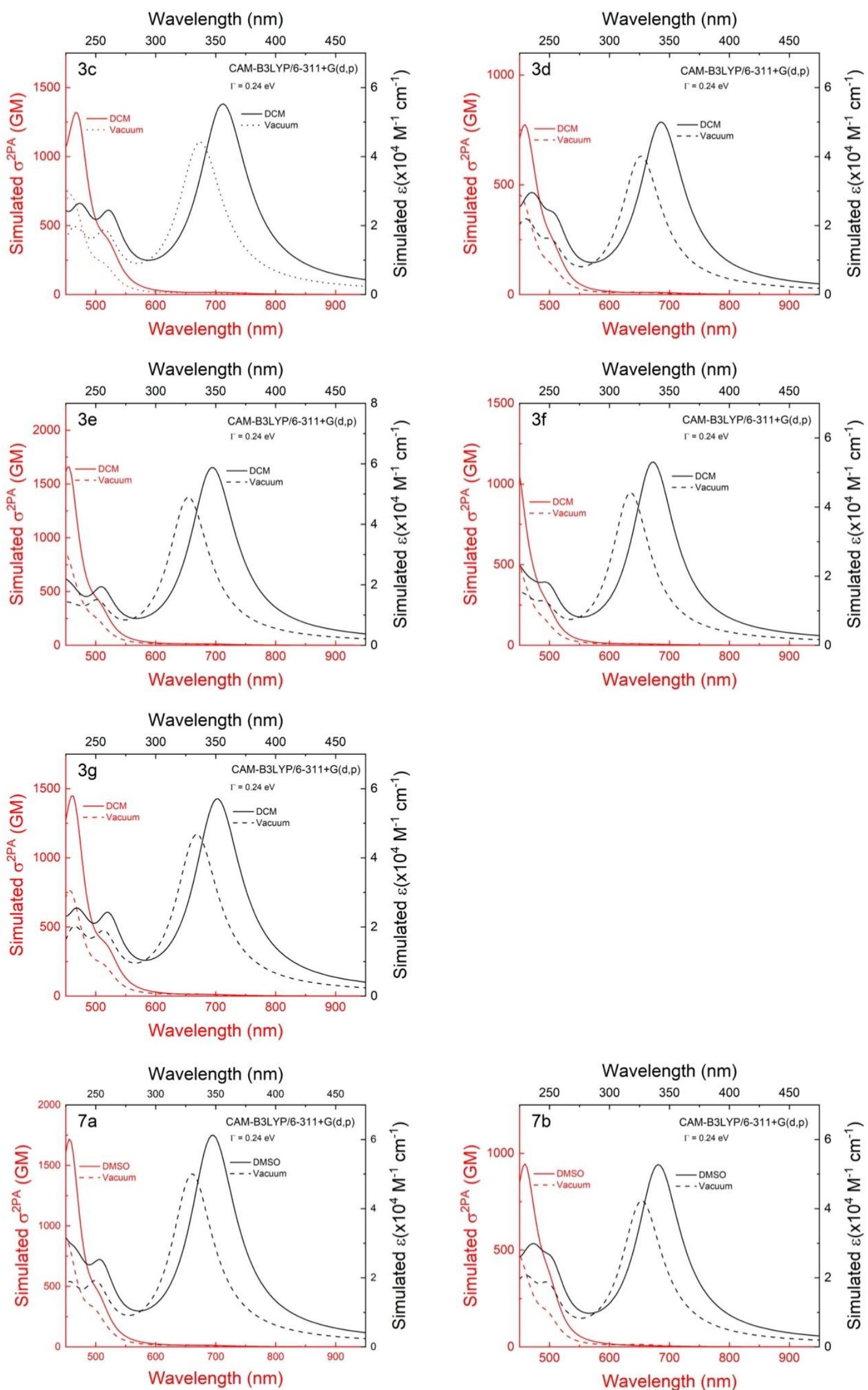

Figure S9: Simulated one-photon absorption (black lines) and two-photon absorption (red lines) in gas-phase (dashed 
line) and solvent media (solid lines).
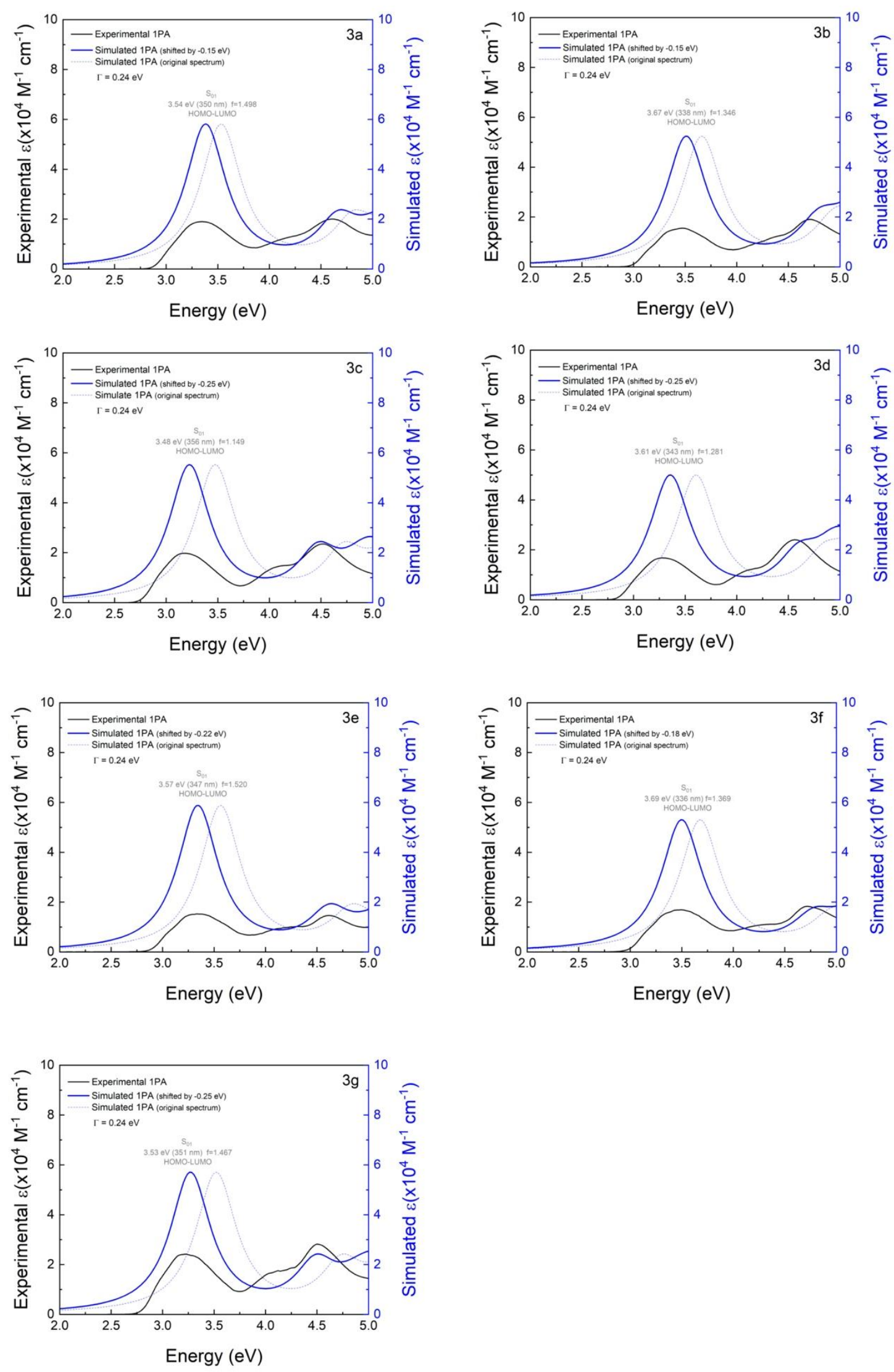

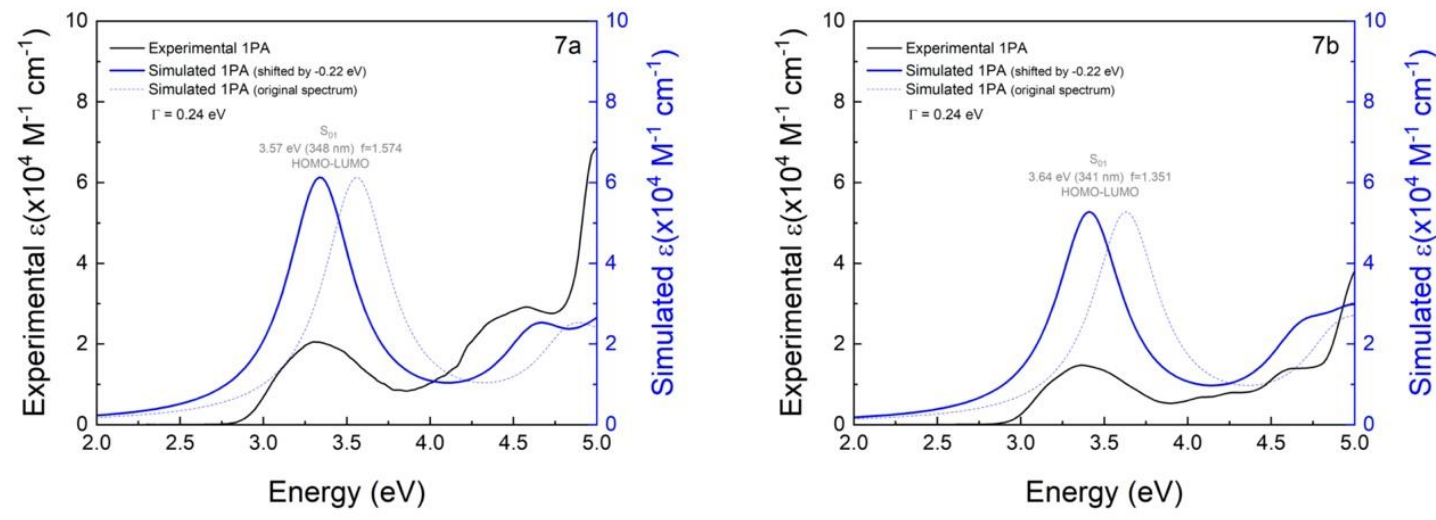

Figure S10: One-photon absorption simulated and experimental spectra of all studied samples in solvent media. Simulated (blue solid and dashed lines, right axis) and experimental (black lines, left axis) 1PA. The solid blue lines represent the simulate 1PA spectra plotted with a redshift between $0.15 \mathrm{eV}$ to $0.25 \mathrm{eV}$. The blue dashed lines are the original simulated 1PA spectra. The value of the damping constant $(\Gamma)$ used in all simulations corresponds to $0.24 \mathrm{eV}$.

\section{b) Optimized geometries}

\begin{tabular}{|c|c|c|c|c|c|c|c|}
\hline \multicolumn{4}{|c|}{ 3a in Vacuum } & \multicolumn{4}{|c|}{$3 \mathrm{a}$ in DCM } \\
\hline \multicolumn{8}{|c|}{ CAM-B3LYP/6-311+G(d,p) } \\
\hline $\mathrm{N}$ & 6.92873 & 6.20900 & -0.10763 & $\mathrm{~N}$ & 6.71811 & 6.42377 & -0.04974 \\
\hline $\mathrm{C}$ & 6.11325 & 5.40181 & -0.17012 & $\mathrm{C}$ & 5.93848 & 5.58198 & -0.12404 \\
\hline $\mathrm{C}$ & 5.09967 & 4.39397 & -0.24691 & $\mathrm{C}$ & 4.96917 & 4.53391 & -0.21624 \\
\hline $\mathrm{C}$ & 3.80362 & 4.73099 & -0.63356 & $\mathrm{C}$ & 3.60892 & 4.83853 & -0.21438 \\
\hline $\mathrm{C}$ & 2.83107 & 3.75057 & -0.70099 & $\mathrm{C}$ & 2.68153 & 3.81641 & -0.30224 \\
\hline $\mathrm{C}$ & 5.40308 & 3.06962 & 0.07674 & $\mathrm{C}$ & 5.38741 & 3.20316 & -0.30552 \\
\hline $\mathrm{C}$ & 4.42463 & 2.09858 & 0.00430 & $\mathrm{C}$ & 4.45197 & 2.19271 & -0.39275 \\
\hline C & 3.12284 & 2.41707 & -0.39939 & $\mathrm{C}$ & 3.08105 & 2.47934 & -0.39446 \\
\hline $\mathrm{C}$ & 2.05840 & 1.41829 & -0.50955 & $\mathrm{C}$ & 2.04550 & 1.44846 & -0.48643 \\
\hline $\mathrm{C}$ & 2.23176 & 0.09578 & -0.58484 & $\mathrm{C}$ & 2.24278 & 0.13314 & -0.61788 \\
\hline $\mathrm{C}$ & 1.12125 & -0.83525 & -0.67777 & $\mathrm{C}$ & 1.14669 & -0.81732 & -0.69513 \\
\hline $\mathrm{N}$ & -0.15073 & -0.51746 & -0.61582 & $\mathrm{~N}$ & -0.13223 & -0.52657 & -0.59986 \\
\hline $\mathrm{C}$ & -0.81846 & -1.70536 & -0.75074 & C & -0.77805 & -1.72704 & -0.73531 \\
\hline $\mathrm{C}$ & -2.18832 & -2.03778 & -0.76389 & $\mathrm{C}$ & -2.14289 & -2.08484 & -0.72338 \\
\hline $\mathrm{N}$ & -3.14199 & -1.10001 & -0.61210 & $\mathrm{~N}$ & -3.11054 & -1.17151 & -0.54240 \\
\hline $\mathrm{C}$ & -4.56903 & -1.38081 & -0.69467 & $\mathrm{C}$ & -4.53617 & -1.47946 & -0.57668 \\
\hline $\mathrm{C}$ & -5.36969 & -0.23480 & -0.13606 & $\mathrm{C}$ & -5.34182 & -0.31053 & -0.07629 \\
\hline C & -5.32549 & 0.06778 & 1.22827 & $\mathrm{C}$ & -5.39358 & -0.01220 & 1.28831 \\
\hline $\mathrm{C}$ & -6.05199 & 1.11692 & 1.75023 & $\mathrm{C}$ & -6.11974 & 1.06454 & 1.75556 \\
\hline C & -6.85202 & 1.89878 & 0.91447 & $\mathrm{C}$ & -6.82057 & 1.87737 & 0.86160 \\
\hline $\mathrm{O}$ & -7.53150 & 2.90679 & 1.51967 & $\mathrm{O}$ & -7.50874 & 2.91084 & 1.41229 \\
\hline $\mathrm{C}$ & -8.36248 & 3.73037 & 0.72691 & $\mathrm{C}$ & -8.24613 & 3.76639 & 0.55225 \\
\hline $\mathrm{C}$ & -6.91056 & 1.61364 & -0.44315 & $\mathrm{C}$ & -6.78287 & 1.59553 & -0.49864 \\
\hline $\mathrm{C}$ & -6.16626 & 0.55123 & -0.95224 & $\mathrm{C}$ & -6.04250 & 0.50550 & -0.95118 \\
\hline $\mathrm{N}$ & -2.53002 & -3.32029 & -0.92247 & $\mathrm{~N}$ & -2.46101 & -3.37540 & -0.89240 \\
\hline $\mathrm{C}$ & -1.56038 & -4.23376 & -1.05062 & $\mathrm{C}$ & -1.47491 & -4.26641 & -1.05553 \\
\hline $\mathrm{N}$ & -0.24932 & -4.04729 & -1.04850 & $\mathrm{~N}$ & -0.16865 & -4.05244 & -1.08058 \\
\hline $\mathrm{C}$ & 0.06831 & -2.76052 & -0.89506 & $\mathrm{C}$ & 0.12866 & -2.75890 & -0.91524 \\
\hline $\mathrm{N}$ & 1.31793 & -2.19762 & -0.84644 & $\mathrm{~N}$ & 1.36581 & -2.17006 & -0.88803 \\
\hline $\mathrm{C}$ & 2.56654 & -2.92718 & -1.00338 & $\mathrm{C}$ & 2.63308 & -2.86851 & -1.04823 \\
\hline $\mathrm{C}$ & 3.43000 & -2.95910 & 0.23794 & $\mathrm{C}$ & 3.44784 & -2.97726 & 0.22264 \\
\hline $\mathrm{C}$ & 4.81474 & -2.91401 & 0.11730 & $\mathrm{C}$ & 2.84344 & -3.07001 & 1.47234 \\
\hline $\mathrm{C}$ & 5.62601 & -2.99109 & 1.24224 & $\mathrm{C}$ & 3.61580 & -3.21481 & 2.61746 \\
\hline $\mathrm{C}$ & 5.05626 & -3.10862 & 2.50154 & $\mathrm{C}$ & 5.00048 & -3.27179 & 2.52517 \\
\hline $\mathrm{C}$ & 3.67369 & -3.15120 & 2.62887 & $\mathrm{C}$ & 5.60961 & -3.18039 & 1.28037 \\
\hline $\mathrm{C}$ & 2.86511 & -3.07832 & 1.50442 & $\mathrm{C}$ & 4.83624 & -3.03115 & 0.13675 \\
\hline $\mathrm{H}$ & 3.56564 & 5.75859 & -0.87631 & $\mathrm{H}$ & 3.28359 & 5.86824 & -0.14385 \\
\hline $\mathrm{H}$ & 1.82450 & 4.01723 & -1.00085 & $\mathrm{H}$ & 1.62502 & 4.05571 & -0.29946 \\
\hline $\mathrm{H}$ & 6.40565 & 2.81347 & 0.39455 & $\mathrm{H}$ & 6.44402 & 2.96912 & -0.30407 \\
\hline $\mathrm{H}$ & 4.67097 & 1.08306 & 0.28752 & $\mathrm{H}$ & 4.79858 & 1.16970 & -0.45656 \\
\hline $\mathrm{H}$ & 1.04217 & 1.79708 & -0.55212 & $\mathrm{H}$ & 1.02151 & 1.80503 & -0.44349 \\
\hline $\mathrm{H}$ & 3.22608 & -0.33333 & -0.57883 & $\mathrm{H}$ & 3.24259 & -0.27919 & -0.66695 \\
\hline $\mathrm{H}$ & -2.83882 & -0.13946 & -0.56436 & $\mathrm{H}$ & -2.82956 & -0.20482 & -0.47998 \\
\hline
\end{tabular}




\begin{tabular}{crrrrrrr}
\hline H & -4.76076 & -2.30162 & -0.14141 & H & -4.71000 & -2.36136 & 0.04187 \\
H & -4.86050 & -1.57325 & -1.73199 & H & -4.84047 & -1.73838 & -1.59489 \\
H & -4.71078 & -0.53460 & 1.88827 & H & -4.85864 & -0.63838 & 1.99410 \\
H & -6.02419 & 1.35246 & 2.80661 & H & -6.16447 & 1.29371 & 2.81309 \\
H & -8.80117 & 4.45744 & 1.40646 & H & -8.71128 & 4.50953 & 1.19515 \\
H & -9.16071 & 3.15167 & 0.25214 & H & -9.02188 & 3.21376 & 0.01593 \\
H & -7.78691 & 4.25534 & -0.04165 & H & -7.58999 & 4.26646 & -0.16483 \\
H & -7.52268 & 2.20078 & -1.11299 & H & -7.31812 & 2.20565 & -1.21222 \\
H & -6.21845 & 0.33670 & -2.01414 & H & -6.02070 & 0.29318 & -2.01447 \\
H & -1.89855 & -5.25765 & -1.17330 & H & -1.79503 & -5.29542 & -1.18578 \\
H & 3.12663 & -2.50950 & -1.84275 & H & 3.21428 & -2.37979 & -1.83121 \\
H & 2.27388 & -3.94185 & -1.28239 & H & 2.37770 & -3.86419 & -1.41446 \\
H & 5.26567 & -2.82060 & -0.86536 & $\mathrm{H}$ & 1.76395 & -3.02671 & 1.55690 \\
H & 6.70340 & -2.95573 & 1.13320 & $\mathrm{H}$ & 3.13285 & -3.28387 & 3.58485 \\
$\mathrm{H}$ & 5.68590 & -3.16477 & 3.38120 & $\mathrm{H}$ & 5.60187 & -3.38335 & 3.41925 \\
$\mathrm{H}$ & 3.22224 & -3.24393 & 3.60936 & $\mathrm{H}$ & 3.25671 & -3.21929 & 3.50151 \\
$\mathrm{H}$ & 1.78717 & -3.11173 & 1.61058 & $\mathrm{H}$ & 1.77212 & -2.95474 & 1.60773 \\
\hline
\end{tabular}

Table S3: Optimized geometries in vacuum and in solvent medium for compound $3 a$

\begin{tabular}{|c|c|c|c|c|c|c|c|}
\hline \multicolumn{4}{|c|}{$3 \mathrm{~b}$ in Vacuum } & & \multicolumn{2}{|c|}{$3 \mathrm{~b}$ in $\mathrm{DCM}$} & \\
\hline \multicolumn{8}{|c|}{ CAM-B3LYP/6-311+G(d,p) } \\
\hline $\mathrm{F}$ & -7.23652 & -3.79226 & -1.27103 & $\mathrm{~F}$ & -7.01248 & -4.13273 & -1.31882 \\
\hline $\mathrm{C}$ & -6.55343 & -3.83509 & -0.10709 & $\mathrm{C}$ & -6.49306 & -3.91219 & -0.08836 \\
\hline $\mathrm{C}$ & -5.30209 & -3.01390 & -0.16522 & $\mathrm{C}$ & -5.26590 & -3.05838 & -0.14140 \\
\hline $\mathrm{C}$ & -4.09120 & -3.60509 & -0.49285 & $\mathrm{C}$ & -4.00929 & -3.64393 & -0.18962 \\
\hline $\mathrm{C}$ & -2.95049 & -2.82461 & -0.58468 & $\mathrm{C}$ & -2.88452 & -2.84023 & -0.28331 \\
\hline $\mathrm{C}$ & -5.36711 & -1.64456 & 0.07245 & $\mathrm{C}$ & -5.39535 & -1.67303 & -0.18841 \\
\hline $\mathrm{C}$ & -4.22581 & -0.87216 & -0.02245 & $\mathrm{C}$ & -4.26931 & -0.87850 & -0.28280 \\
\hline $\mathrm{C}$ & -2.99625 & -1.44640 & -0.36431 & $\mathrm{C}$ & -2.99166 & -1.44830 & -0.33627 \\
\hline $\mathrm{C}$ & -1.76074 & -0.67009 & -0.49098 & $\mathrm{C}$ & -1.76257 & -0.65776 & -0.44058 \\
\hline $\mathrm{C}$ & -1.66986 & 0.66083 & -0.55688 & $\mathrm{C}$ & -1.67779 & 0.66817 & -0.58279 \\
\hline $\mathrm{C}$ & -0.39729 & 1.35270 & -0.66622 & $\mathrm{C}$ & -0.40495 & 1.36353 & -0.67410 \\
\hline $\mathrm{N}$ & 0.78788 & 0.79183 & -0.60242 & $\mathrm{~N}$ & 0.78384 & 0.81046 & -0.57368 \\
\hline $\mathrm{C}$ & 1.67528 & 1.82392 & -0.75778 & $\mathrm{C}$ & 1.66887 & 1.84489 & -0.72983 \\
\hline $\mathrm{C}$ & 3.08357 & 1.88073 & -0.78238 & $\mathrm{C}$ & 3.07829 & 1.90608 & -0.72567 \\
\hline $\mathrm{N}$ & 3.83575 & 0.77589 & -0.62205 & $\mathrm{~N}$ & 3.83240 & 0.81206 & -0.53038 \\
\hline $\mathrm{C}$ & 5.28923 & 0.77002 & -0.71238 & $\mathrm{C}$ & 5.29075 & 0.81261 & -0.57361 \\
\hline $\mathrm{C}$ & 5.85256 & -0.50659 & -0.14716 & $\mathrm{C}$ & 5.83426 & -0.49970 & -0.07541 \\
\hline $\mathrm{C}$ & 5.75949 & -0.78303 & 1.22040 & $\mathrm{C}$ & 5.83914 & -0.79720 & 1.29031 \\
\hline $\mathrm{C}$ & 6.26969 & -1.94984 & 1.74870 & $\mathrm{C}$ & 6.32195 & -2.00373 & 1.75540 \\
\hline $\mathrm{C}$ & 6.89480 & -2.88060 & 0.91626 & $\mathrm{C}$ & 6.81837 & -2.95199 & 0.85802 \\
\hline $\mathrm{O}$ & 7.36781 & -3.99723 & 1.52740 & $\mathrm{O}$ & 7.27416 & -4.10799 & 1.40654 \\
\hline $\mathrm{C}$ & 8.01807 & -4.97260 & 0.73843 & $\mathrm{C}$ & 7.79388 & -5.10716 & 0.54232 \\
\hline $\mathrm{C}$ & 6.99810 & -2.62423 & -0.44433 & $\mathrm{C}$ & 6.82430 & -2.67338 & -0.50345 \\
\hline $\mathrm{C}$ & 6.47322 & -1.44071 & -0.95985 & $\mathrm{C}$ & 6.33052 & -1.45093 & -0.95374 \\
\hline $\mathrm{N}$ & 3.66949 & 3.06838 & -0.96351 & $\mathrm{~N}$ & 3.66174 & 3.09702 & -0.91772 \\
\hline $\mathrm{C}$ & 2.89714 & 4.15260 & -1.10354 & $\mathrm{C}$ & 2.88578 & 4.17375 & -1.09506 \\
\hline $\mathrm{N}$ & 1.57517 & 4.22710 & -1.09431 & $\mathrm{~N}$ & 1.56368 & 4.24048 & -1.11488 \\
\hline $\mathrm{C}$ & 1.01264 & 3.02981 & -0.91766 & $\mathrm{C}$ & 1.00044 & 3.04192 & -0.92706 \\
\hline $\mathrm{N}$ & -0.32340 & 2.72382 & -0.85649 & $\mathrm{~N}$ & -0.33328 & 2.72869 & -0.88962 \\
\hline $\mathrm{C}$ & -1.40379 & 3.68412 & -1.01635 & $\mathrm{C}$ & -1.42368 & 3.67685 & -1.06564 \\
\hline $\mathrm{C}$ & -2.21718 & 3.92105 & 0.23691 & $\mathrm{C}$ & -2.19189 & 3.98361 & 0.20186 \\
\hline $\mathrm{C}$ & -3.58537 & 4.15141 & 0.13688 & $\mathrm{C}$ & -3.53775 & 4.32841 & 0.11434 \\
\hline $\mathrm{C}$ & -4.34306 & 4.41661 & 1.27018 & $\mathrm{C}$ & -4.25665 & 4.66399 & 1.25402 \\
\hline $\mathrm{C}$ & -3.73737 & 4.44802 & 2.51799 & $\mathrm{C}$ & -3.63634 & 4.65237 & 2.49659 \\
\hline $\mathrm{C}$ & -2.37212 & 4.21617 & 2.62472 & $\mathrm{C}$ & -2.29480 & 4.30521 & 2.59064 \\
\hline $\mathrm{C}$ & -1.61601 & 3.95584 & 1.49117 & $\mathrm{C}$ & -1.57597 & 3.97416 & 1.44936 \\
\hline $\mathrm{F}$ & -7.39922 & -3.40199 & 0.84559 & $\mathrm{~F}$ & -7.47802 & -3.34888 & 0.63728 \\
\hline $\mathrm{F}$ & -6.30259 & -5.13148 & 0.14269 & $\mathrm{~F}$ & -6.25659 & -5.12446 & 0.44376 \\
\hline $\mathrm{H}$ & -4.03786 & -4.67190 & -0.66634 & $\mathrm{H}$ & -3.90529 & -4.72015 & -0.14757 \\
\hline $\mathrm{H}$ & -2.00429 & -3.28942 & -0.83561 & $\mathrm{H}$ & -1.90359 & -3.29905 & -0.31589 \\
\hline $\mathrm{H}$ & -6.30983 & -1.18907 & 0.34776 & $\mathrm{H}$ & -6.37593 & -1.21680 & -0.14208 \\
\hline $\mathrm{H}$ & -4.28972 & 0.18709 & 0.19208 & $\mathrm{H}$ & -4.39196 & 0.19639 & -0.30667 \\
\hline $\mathrm{H}$ & -0.83866 & -1.23989 & -0.54880 & $\mathrm{H}$ & -0.83635 & -1.22204 & -0.40246 \\
\hline $\mathrm{H}$ & -2.55738 & 1.28081 & -0.52615 & $\mathrm{H}$ & -2.56785 & 1.28252 & -0.63361 \\
\hline $\mathrm{H}$ & 3.34990 & -0.10523 & -0.55507 & $\mathrm{H}$ & 3.35435 & -0.07252 & -0.45135 \\
\hline $\mathrm{H}$ & 5.66202 & 1.63927 & -0.16804 & $\mathrm{H}$ & 5.65122 & 1.63906 & 0.04114 \\
\hline $\mathrm{H}$ & 5.60723 & 0.89332 & -1.75251 & $\mathrm{H}$ & 5.63578 & 1.00005 & -1.59439 \\
\hline $\mathrm{H}$ & 5.27936 & -0.06606 & 1.87747 & $\mathrm{H}$ & 5.46138 & -0.06782 & 1.99860 \\
\hline $\mathrm{H}$ & 6.20359 & -2.16654 & 2.80742 & $\mathrm{H}$ & 6.33007 & -2.23340 & 2.81373 \\
\hline $\mathrm{H}$ & 8.31296 & -5.76501 & 1.42259 & $\mathrm{H}$ & 8.09463 & -5.93181 & 1.18375 \\
\hline
\end{tabular}




\begin{tabular}{rrrrrrrr}
\hline $\mathrm{H}$ & 8.90971 & -4.56335 & 0.25361 & $\mathrm{H}$ & 8.66374 & -4.73963 & -0.00818 \\
$\mathrm{H}$ & 7.34643 & -5.38317 & -0.02180 & $\mathrm{H}$ & 7.03386 & -5.45404 & -0.16258 \\
$\mathrm{H}$ & 7.47753 & -3.32625 & -1.11178 & $\mathrm{H}$ & 7.20608 & -3.38702 & -1.21966 \\
$\mathrm{H}$ & 6.55848 & -1.24983 & -2.02412 & $\mathrm{H}$ & 6.34067 & -1.24303 & -2.01810 \\
$\mathrm{H}$ & 3.42940 & 5.08772 & -1.24512 & $\mathrm{H}$ & 3.41565 & 5.10944 & -1.24355 \\
$\mathrm{H}$ & -2.05433 & 3.36275 & -1.83245 & $\mathrm{H}$ & -2.09829 & 3.30666 & -1.83866 \\
$\mathrm{H}$ & -0.92156 & 4.61201 & -1.33120 & $\mathrm{H}$ & -0.96354 & 4.58747 & -1.45269 \\
$\mathrm{H}$ & -4.06502 & 4.12508 & -0.83627 & $\mathrm{H}$ & -4.02928 & 4.33357 & -0.85273 \\
$\mathrm{H}$ & -5.40777 & 4.59464 & 1.17714 & $\mathrm{H}$ & -5.30396 & 4.92883 & 1.17105 \\
$\mathrm{H}$ & -4.32617 & 4.65036 & 3.40443 & $\mathrm{H}$ & -4.19638 & 4.90909 & 3.38764 \\
$\mathrm{H}$ & -1.89249 & 4.23951 & 3.59588 & $\mathrm{H}$ & -1.80374 & 4.29203 & 3.55632 \\
$\mathrm{H}$ & -0.55094 & 3.77754 & 1.58205 & $\mathrm{H}$ & -0.52981 & 3.70488 & 1.53509 \\
\hline
\end{tabular}

Table S4: Optimized geometries in vacuum and in solvent medium for compound $3 \mathrm{~b}$

\begin{tabular}{|c|c|c|c|c|c|c|c|}
\hline \multicolumn{4}{|c|}{$3 c$ in Vacuum } & \multicolumn{4}{|c|}{$3 c$ in DCM } \\
\hline \multicolumn{8}{|c|}{ CAM-B3LYP/6-311+G(d,p) } \\
\hline $\mathrm{N}$ & -8.23709 & -2.79071 & 0.00930 & $\mathrm{~N}$ & -8.28907 & -2.72670 & 0.00890 \\
\hline $\mathrm{C}$ & -7.12025 & -2.52253 & -0.02651 & $\mathrm{C}$ & -7.17021 & -2.46396 & -0.02576 \\
\hline $\mathrm{C}$ & -5.72941 & -2.18728 & -0.07221 & $\mathrm{C}$ & -5.77851 & -2.13628 & -0.06996 \\
\hline $\mathrm{C}$ & -4.76792 & -3.19250 & -0.15755 & $\mathrm{C}$ & -4.82082 & -3.14751 & -0.00669 \\
\hline $\mathrm{C}$ & -3.42693 & -2.85762 & -0.19807 & $\mathrm{C}$ & -3.47831 & -2.81881 & -0.04835 \\
\hline $\mathrm{C}$ & -5.32899 & -0.84995 & -0.02190 & $\mathrm{C}$ & -5.37895 & -0.80068 & -0.17025 \\
\hline $\mathrm{C}$ & -3.98768 & -0.52743 & -0.06329 & $\mathrm{C}$ & -4.03599 & -0.48605 & -0.21163 \\
\hline $\mathrm{C}$ & -3.00938 & -1.52387 & -0.16325 & $\mathrm{C}$ & -3.05973 & -1.48889 & -0.15922 \\
\hline $\mathrm{C}$ & -1.57677 & -1.23103 & -0.22634 & $\mathrm{C}$ & -1.62333 & -1.21010 & -0.21137 \\
\hline $\mathrm{C}$ & -1.02808 & -0.03500 & -0.45787 & $\mathrm{C}$ & -1.05716 & -0.03148 & -0.48849 \\
\hline $\mathrm{C}$ & 0.40818 & 0.17787 & -0.48628 & $\mathrm{C}$ & 0.38259 & 0.16181 & -0.51319 \\
\hline $\mathrm{N}$ & 1.31849 & -0.71243 & -0.17588 & $\mathrm{~N}$ & 1.28283 & -0.73937 & -0.19799 \\
\hline $\mathrm{C}$ & 2.52446 & -0.07525 & -0.34911 & $\mathrm{C}$ & 2.49705 & -0.11803 & -0.37207 \\
\hline $\mathrm{C}$ & 3.87751 & -0.46592 & -0.18992 & $\mathrm{C}$ & 3.84521 & -0.53093 & -0.21101 \\
\hline $\mathrm{N}$ & 4.27373 & -1.68212 & 0.21552 & $\mathrm{~N}$ & 4.22380 & -1.74636 & 0.20148 \\
\hline $\mathrm{C}$ & 5.69038 & -2.04101 & 0.32728 & $\mathrm{C}$ & 5.63518 & -2.13444 & 0.30971 \\
\hline $\mathrm{C}$ & 5.65139 & -3.54956 & 0.56398 & $\mathrm{C}$ & 5.56801 & -3.63807 & 0.56569 \\
\hline $\mathrm{C}$ & 4.32373 & -3.76065 & 1.29410 & $\mathrm{C}$ & 4.24664 & -3.81112 & 1.31528 \\
\hline $\mathrm{C}$ & 3.38195 & -2.77982 & 0.59910 & $\mathrm{C}$ & 3.31589 & -2.82244 & 0.61776 \\
\hline $\mathrm{N}$ & 4.82915 & 0.44311 & -0.46703 & $\mathrm{~N}$ & 4.81000 & 0.36743 & -0.49851 \\
\hline $\mathrm{C}$ & 4.47445 & 1.66175 & -0.87273 & $\mathrm{C}$ & 4.47007 & 1.58851 & -0.91002 \\
\hline $\mathrm{N}$ & 3.25366 & 2.14450 & -1.05299 & $\mathrm{~N}$ & 3.25603 & 2.08811 & -1.08945 \\
\hline $\mathrm{C}$ & 2.32095 & 1.23339 & -0.77659 & $\mathrm{C}$ & 2.30737 & 1.19042 & -0.80641 \\
\hline $\mathrm{N}$ & 0.96235 & 1.39073 & -0.86018 & $\mathrm{~N}$ & 0.95251 & 1.36374 & -0.89201 \\
\hline $\mathrm{C}$ & 0.29483 & 2.60155 & -1.31064 & $\mathrm{C}$ & 0.28903 & 2.58424 & -1.32626 \\
\hline $\mathrm{C}$ & -0.49274 & 3.31473 & -0.23396 & $\mathrm{C}$ & -0.40822 & 3.34011 & -0.21591 \\
\hline $\mathrm{C}$ & -0.02163 & 3.39036 & 1.07309 & $\mathrm{C}$ & 0.09466 & 3.35577 & 1.08150 \\
\hline $\mathrm{C}$ & -0.73710 & 4.08430 & 2.03792 & $\mathrm{C}$ & -0.53852 & 4.09499 & 2.07188 \\
\hline $\mathrm{C}$ & -1.93032 & 4.71378 & 1.70747 & $\mathrm{C}$ & -1.67985 & 4.82931 & 1.77535 \\
\hline $\mathrm{C}$ & -2.40499 & 4.64418 & 0.40567 & $\mathrm{C}$ & -2.18654 & 4.81784 & 0.48245 \\
\hline $\mathrm{C}$ & -1.68964 & 3.94518 & -0.55801 & $\mathrm{C}$ & -1.55465 & 4.07435 & -0.50594 \\
\hline $\mathrm{H}$ & -5.07627 & -4.22952 & -0.19038 & $\mathrm{H}$ & -5.12903 & -4.18162 & 0.07544 \\
\hline $\mathrm{H}$ & -2.68321 & -3.64293 & -0.26435 & $\mathrm{H}$ & -2.73639 & -3.60661 & 0.00154 \\
\hline $\mathrm{H}$ & -6.07655 & -0.07121 & 0.05871 & $\mathrm{H}$ & -6.12404 & -0.01668 & -0.20944 \\
\hline $\mathrm{H}$ & -3.69738 & 0.51356 & 0.00079 & $\mathrm{H}$ & -3.74755 & 0.55502 & -0.27468 \\
\hline $\mathrm{H}$ & -0.90651 & -2.07284 & -0.08423 & $\mathrm{H}$ & -0.96995 & -2.05429 & -0.01639 \\
\hline $\mathrm{H}$ & -1.64891 & 0.83536 & -0.63231 & $\mathrm{H}$ & -1.66431 & 0.83740 & -0.70984 \\
\hline $\mathrm{H}$ & 6.14795 & -1.50522 & 1.16499 & $\mathrm{H}$ & 6.10705 & -1.60064 & 1.14040 \\
\hline $\mathrm{H}$ & 6.22936 & -1.75161 & -0.57423 & $\mathrm{H}$ & 6.17429 & -1.87222 & -0.59976 \\
\hline $\mathrm{H}$ & 6.51488 & -3.90120 & 1.12883 & $\mathrm{H}$ & 6.43122 & -3.99753 & 1.12531 \\
\hline $\mathrm{H}$ & 5.63990 & -4.07769 & -0.39325 & $\mathrm{H}$ & 5.53346 & -4.17695 & -0.38462 \\
\hline $\mathrm{H}$ & 4.42839 & -3.49119 & 2.34852 & $\mathrm{H}$ & 4.36973 & -3.52884 & 2.36405 \\
\hline $\mathrm{H}$ & 3.96076 & -4.78751 & 1.24484 & $\mathrm{H}$ & 3.86121 & -4.82997 & 1.28276 \\
\hline $\mathrm{H}$ & 2.57574 & -2.42153 & 1.23649 & $\mathrm{H}$ & 2.53047 & -2.43680 & 1.26437 \\
\hline $\mathrm{H}$ & 2.92157 & -3.22510 & -0.28926 & $\mathrm{H}$ & 2.83614 & -3.27227 & -0.25692 \\
\hline $\mathrm{H}$ & 5.29471 & 2.34220 & -1.07940 & $\mathrm{H}$ & 5.29930 & 2.25665 & -1.12299 \\
\hline $\mathrm{H}$ & -0.35385 & 2.36264 & -2.15606 & $\mathrm{H}$ & -0.41642 & 2.34415 & -2.12269 \\
\hline $\mathrm{H}$ & 1.09134 & 3.24871 & -1.68376 & $\mathrm{H}$ & 1.07088 & 3.20474 & -1.76690 \\
\hline $\mathrm{H}$ & 0.90997 & 2.90382 & 1.33744 & $\mathrm{H}$ & 0.98450 & 2.78662 & 1.32379 \\
\hline $\mathrm{H}$ & -0.35994 & 4.13548 & 3.05228 & $\mathrm{H}$ & -0.13768 & 4.09755 & 3.07847 \\
\hline $\mathrm{H}$ & -2.48775 & 5.25488 & 2.46238 & $\mathrm{H}$ & -2.17353 & 5.40479 & 2.54908 \\
\hline $\mathrm{H}$ & -3.33530 & 5.13120 & 0.13863 & $\mathrm{H}$ & -3.07882 & 5.38370 & 0.24253 \\
\hline $\mathrm{H}$ & -2.06692 & 3.89201 & -1.57419 & $\mathrm{H}$ & -1.95864 & 4.06443 & -1.51274 \\
\hline
\end{tabular}

Table S5: Optimized geometries in vacuum and in solvent medium for compound $3 \mathrm{c}$ 


\begin{tabular}{|c|c|c|c|c|c|c|c|}
\hline \multicolumn{4}{|c|}{$3 d$ in Vacuum } & \multicolumn{4}{|c|}{$3 \mathrm{~d}$ in $\mathrm{DCM}$} \\
\hline \multicolumn{8}{|c|}{ CAM-B3LYP/6-311+G(d,p) } \\
\hline $\mathrm{F}$ & 7.21724 & -1.42574 & 1.27335 & $\mathrm{~F}$ & 7.26741 & -1.24861 & 1.26209 \\
\hline $\mathrm{C}$ & 6.79001 & -1.42440 & -0.00742 & $\mathrm{C}$ & 6.82007 & -1.38362 & -0.00827 \\
\hline $\mathrm{C}$ & 5.30273 & -1.27478 & -0.10019 & $\mathrm{C}$ & 5.33421 & -1.23886 & -0.10070 \\
\hline $\mathrm{C}$ & 4.73463 & -0.00641 & -0.03528 & $\mathrm{C}$ & 4.77160 & 0.03060 & -0.19891 \\
\hline $\mathrm{C}$ & 3.36130 & 0.13806 & -0.07086 & $\mathrm{C}$ & 3.39805 & 0.17461 & -0.23707 \\
\hline $\mathrm{C}$ & 4.49062 & -2.39523 & -0.19167 & $\mathrm{C}$ & 4.51919 & -2.35969 & -0.03264 \\
\hline $\mathrm{C}$ & 3.11433 & -2.24281 & -0.22690 & $\mathrm{C}$ & 3.14280 & -2.20711 & -0.07123 \\
\hline $\mathrm{C}$ & 2.52386 & -0.97814 & -0.17763 & $\mathrm{C}$ & 2.55724 & -0.94318 & -0.18020 \\
\hline $\mathrm{C}$ & 1.06391 & -0.87685 & -0.23730 & $\mathrm{C}$ & 1.09574 & -0.85053 & -0.22909 \\
\hline $\mathrm{C}$ & 0.36096 & 0.23625 & -0.46308 & $\mathrm{C}$ & 0.37922 & 0.24608 & -0.49316 \\
\hline $\mathrm{C}$ & -1.09133 & 0.25645 & -0.48823 & $\mathrm{C}$ & -1.07411 & 0.24794 & -0.51208 \\
\hline $\mathrm{N}$ & -1.87536 & -0.74622 & -0.17638 & $\mathrm{~N}$ & -1.84693 & -0.76450 & -0.19689 \\
\hline $\mathrm{C}$ & -3.15562 & -0.27426 & -0.34837 & $\mathrm{C}$ & -3.13341 & -0.30788 & -0.36562 \\
\hline $\mathrm{C}$ & -4.44448 & -0.84051 & -0.18731 & $\mathrm{C}$ & -4.41486 & -0.89525 & -0.20373 \\
\hline $\mathrm{N}$ & -4.67544 & -2.09871 & 0.21885 & $\mathrm{~N}$ & -4.62976 & -2.15240 & 0.20233 \\
\hline $\mathrm{C}$ & -3.64519 & -3.06848 & 0.60010 & $\mathrm{C}$ & -3.58804 & -3.10354 & 0.60897 \\
\hline $\mathrm{C}$ & -4.44724 & -4.16588 & 1.29612 & $\mathrm{C}$ & -4.37926 & -4.20544 & 1.30873 \\
\hline $\mathrm{C}$ & -5.79254 & -4.13244 & 0.56852 & $\mathrm{C}$ & -5.71474 & -4.20476 & 0.56413 \\
\hline $\mathrm{C}$ & -6.03163 & -2.64224 & 0.33290 & $\mathrm{C}$ & -5.97798 & -2.72229 & 0.31029 \\
\hline $\mathrm{N}$ & -5.50881 & -0.06586 & -0.46307 & $\mathrm{~N}$ & -5.49056 & -0.13026 & -0.48390 \\
\hline $\mathrm{C}$ & -5.31924 & 1.18889 & -0.86950 & $\mathrm{C}$ & -5.31560 & 1.12633 & -0.89157 \\
\hline $\mathrm{N}$ & -4.17339 & 1.82895 & -1.05159 & $\mathrm{~N}$ & -4.17824 & 1.78131 & -1.07327 \\
\hline $\mathrm{C}$ & -3.12776 & 1.04942 & -0.77631 & $\mathrm{C}$ & -3.11888 & 1.01528 & -0.79588 \\
\hline $\mathrm{N}$ & -1.80187 & 1.38515 & -0.86159 & $\mathrm{~N}$ & -1.79866 & 1.36558 & -0.88491 \\
\hline $\mathrm{C}$ & -1.30075 & 2.67307 & -1.31357 & $\mathrm{C}$ & -1.30282 & 2.66249 & -1.32159 \\
\hline $\mathrm{C}$ & -0.61942 & 3.48788 & -0.23649 & $\mathrm{C}$ & -0.69586 & 3.49877 & -0.21606 \\
\hline $\mathrm{C}$ & -1.10402 & 3.50649 & 1.06770 & $\mathrm{C}$ & -1.19142 & 3.45651 & 1.08361 \\
\hline $\mathrm{C}$ & -0.49096 & 4.29162 & 2.03306 & $\mathrm{C}$ & -0.64632 & 4.26722 & 2.07033 \\
\hline $\mathrm{C}$ & 0.61180 & 5.07024 & 1.70605 & $\mathrm{C}$ & 0.39843 & 5.13155 & 1.76791 \\
\hline $\mathrm{C}$ & 1.09913 & 5.05811 & 0.40717 & $\mathrm{C}$ & 0.89709 & 5.17860 & 0.47277 \\
\hline $\mathrm{C}$ & 0.48675 & 4.26798 & -0.55706 & $\mathrm{C}$ & 0.35355 & 4.36381 & -0.51205 \\
\hline $\mathrm{F}$ & 7.43857 & -0.41849 & -0.62270 & $\mathrm{~F}$ & 7.47165 & -0.45503 & -0.73446 \\
\hline $\mathrm{F}$ & 7.22278 & -2.57195 & -0.55718 & $\mathrm{~F}$ & 7.24890 & -2.58603 & -0.43267 \\
\hline $\mathrm{H}$ & 5.37059 & 0.86611 & 0.04446 & $\mathrm{H}$ & 5.40761 & 0.90526 & -0.24647 \\
\hline $\mathrm{H}$ & 2.93591 & 1.13105 & 0.00080 & $\mathrm{H}$ & 2.98052 & 1.17081 & -0.30368 \\
\hline $\mathrm{H}$ & 4.93182 & -3.38213 & -0.24124 & $\mathrm{H}$ & 4.95364 & -3.34773 & 0.04504 \\
\hline $\mathrm{H}$ & 2.48192 & -3.11959 & -0.30243 & $\mathrm{H}$ & 2.50844 & -3.08398 & -0.02117 \\
\hline $\mathrm{H}$ & 0.51143 & -1.80061 & -0.09704 & $\mathrm{H}$ & 0.55764 & -1.77399 & -0.04059 \\
\hline $\mathrm{H}$ & 0.86055 & 1.18180 & -0.63529 & $\mathrm{H}$ & 0.86541 & 1.18999 & -0.70610 \\
\hline $\mathrm{H}$ & -2.89237 & -2.60674 & 1.23619 & $\mathrm{H}$ & -2.85418 & -2.62150 & 1.25124 \\
\hline $\mathrm{H}$ & -3.13136 & -3.44849 & -0.28929 & $\mathrm{H}$ & -3.06081 & -3.48645 & -0.27031 \\
\hline $\mathrm{H}$ & -4.58479 & -3.91320 & 2.35094 & $\mathrm{H}$ & -4.53383 & -3.94256 & 2.35838 \\
\hline $\mathrm{H}$ & -3.95136 & -5.13552 & 1.24553 & $\mathrm{H}$ & -3.86485 & -5.16557 & 1.27316 \\
\hline $\mathrm{H}$ & -6.60077 & -4.59581 & 1.13461 & $\mathrm{H}$ & -6.52187 & -4.67387 & 1.12630 \\
\hline $\mathrm{H}$ & -5.71280 & -4.65391 & -0.38912 & $\mathrm{H}$ & -5.61394 & -4.73390 & -0.38693 \\
\hline $\mathrm{H}$ & -6.55486 & -2.17239 & 1.17180 & $\mathrm{H}$ & -6.51569 & -2.25605 & 1.14156 \\
\hline $\mathrm{H}$ & -6.60603 & -2.42660 & -0.56743 & $\mathrm{H}$ & -6.54702 & -2.53208 & -0.59896 \\
\hline $\mathrm{H}$ & -6.22269 & 1.75464 & -1.07514 & $\mathrm{H}$ & -6.22618 & 1.68062 & -1.09902 \\
\hline $\mathrm{H}$ & -2.17517 & 3.20725 & -1.69109 & $\mathrm{H}$ & -2.16318 & 3.17843 & -1.75073 \\
\hline $\mathrm{H}$ & -0.62281 & 2.52081 & -2.15603 & $\mathrm{H}$ & -0.58150 & 2.51731 & -2.12680 \\
\hline $\mathrm{H}$ & -1.96550 & 2.90327 & 1.32927 & $\mathrm{H}$ & -2.00620 & 2.78581 & 1.33016 \\
\hline $\mathrm{H}$ & -0.87736 & 4.29692 & 3.04522 & $\mathrm{H}$ & -1.04012 & 4.22360 & 3.07876 \\
\hline $\mathrm{H}$ & 1.08954 & 5.68219 & 2.46151 & $\mathrm{H}$ & 0.82379 & 5.76257 & 2.53891 \\
\hline $\mathrm{H}$ & 1.95973 & 5.66103 & 0.14286 & $\mathrm{H}$ & 1.71485 & 5.84600 & 0.22829 \\
\hline $\mathrm{H}$ & 0.87409 & 4.26012 & -1.57080 & $\mathrm{H}$ & 0.75146 & 4.40038 & -1.52058 \\
\hline
\end{tabular}

Table S6: Optimized geometries in vacuum and in solvent medium for compound $3 \mathrm{~d}$

\begin{tabular}{|c|c|c|c|c|c|c|c|}
\hline \multicolumn{4}{|c|}{ 3e in Vacuum } & \multicolumn{4}{|c|}{$3 e$ in DCM } \\
\hline \multicolumn{8}{|c|}{ CAM-B3LYP/6-311+G(d,p) } \\
\hline $\mathrm{N}$ & -8.50862 & -3.08524 & 0.42352 & $\mathrm{~N}$ & -8.53955 & -3.05739 & 0.43211 \\
\hline $\mathrm{C}$ & -7.40445 & -2.78954 & 0.30617 & $\mathrm{C}$ & -7.43512 & -2.76064 & 0.31343 \\
\hline $\mathrm{C}$ & -6.02954 & -2.41918 & 0.15897 & $\mathrm{C}$ & -6.06132 & -2.39029 & 0.16471 \\
\hline $\mathrm{C}$ & -5.05847 & -3.39639 & -0.05094 & $\mathrm{C}$ & -5.07638 & -3.37451 & 0.09670 \\
\hline $\mathrm{C}$ & -3.73282 & -3.02762 & -0.18841 & $\mathrm{C}$ & -3.75163 & -3.00433 & -0.04586 \\
\hline $\mathrm{C}$ & -5.65445 & -1.07574 & 0.23458 & $\mathrm{C}$ & -5.70698 & -1.04023 & 0.09319 \\
\hline $\mathrm{C}$ & -4.32846 & -0.71918 & 0.09503 & $\mathrm{C}$ & -4.38150 & -0.68407 & -0.05003 \\
\hline $\mathrm{C}$ & -3.34222 & -1.68675 & -0.12994 & $\mathrm{C}$ & -3.37936 & -1.65897 & -0.12981 \\
\hline $\mathrm{C}$ & -1.92624 & -1.35694 & -0.29928 & $\mathrm{C}$ & -1.96062 & -1.33618 & -0.29415 \\
\hline $\mathrm{C}$ & -1.42278 & -0.13938 & -0.52007 & $\mathrm{C}$ & -1.44973 & -0.12923 & -0.55537 \\
\hline $\mathrm{C}$ & 0.00232 & 0.10392 & -0.65874 & $\mathrm{C}$ & -0.02249 & 0.10174 & -0.69873 \\
\hline
\end{tabular}




\begin{tabular}{|c|c|c|c|c|c|c|c|}
\hline $\mathrm{N}$ & 0.95274 & -0.78179 & -0.46896 & $\mathrm{~N}$ & 0.92396 & -0.79240 & -0.51542 \\
\hline $\mathrm{C}$ & 2.11843 & -0.10501 & -0.71363 & $\mathrm{C}$ & 2.09352 & -0.12456 & -0.76763 \\
\hline $\mathrm{C}$ & 3.47321 & -0.48993 & -0.67975 & $\mathrm{C}$ & 3.44566 & -0.52311 & -0.73948 \\
\hline $\mathrm{N}$ & 3.83683 & -1.74959 & -0.36424 & $\mathrm{~N}$ & 3.79987 & -1.78330 & -0.42630 \\
\hline $\mathrm{C}$ & 5.20878 & -2.21806 & -0.34604 & $\mathrm{C}$ & 5.16752 & -2.26160 & -0.37405 \\
\hline $\mathrm{C}$ & 5.90747 & -1.91616 & 0.97012 & $\mathrm{C}$ & 5.81371 & -2.00572 & 0.97849 \\
\hline $\mathrm{O}$ & 7.19879 & -2.47113 & 0.90092 & $\mathrm{O}$ & 7.12468 & -2.52471 & 0.93480 \\
\hline $\mathrm{C}$ & 7.97766 & -2.20151 & 2.04076 & $\mathrm{C}$ & 7.83476 & -2.32042 & 2.13835 \\
\hline $\mathrm{N}$ & 4.40158 & 0.42353 & -0.97469 & $\mathrm{~N}$ & 4.37989 & 0.38639 & -1.04238 \\
\hline $\mathrm{C}$ & 4.00792 & 1.66170 & -1.29628 & $\mathrm{C}$ & 3.99322 & 1.62754 & -1.36114 \\
\hline $\mathrm{N}$ & 2.77526 & 2.14140 & -1.36016 & $\mathrm{~N}$ & 2.76400 & 2.11695 & -1.41723 \\
\hline $\mathrm{C}$ & 1.86602 & 1.21332 & -1.05693 & $\mathrm{C}$ & 1.84575 & 1.19541 & -1.10870 \\
\hline $\mathrm{N}$ & 0.50123 & 1.34591 & -1.01911 & $\mathrm{~N}$ & 0.48374 & 1.33747 & -1.06200 \\
\hline $\mathrm{C}$ & -0.22511 & 2.56180 & -1.35128 & $\mathrm{C}$ & -0.24429 & 2.56312 & -1.35917 \\
\hline $\mathrm{C}$ & -0.93476 & 3.20252 & -0.17906 & $\mathrm{C}$ & -0.85550 & 3.23042 & -0.14603 \\
\hline $\mathrm{C}$ & -0.36223 & 3.21982 & 1.08892 & $\mathrm{C}$ & -0.24113 & 3.17796 & 1.10140 \\
\hline $\mathrm{C}$ & -1.00938 & 3.84874 & 2.14250 & $\mathrm{C}$ & -0.79967 & 3.83766 & 2.18815 \\
\hline $\mathrm{C}$ & -2.23466 & 4.47084 & 1.94067 & $\mathrm{C}$ & -1.97693 & 4.55977 & 2.03896 \\
\hline $\mathrm{C}$ & -2.81036 & 4.45921 & 0.67841 & $\mathrm{C}$ & -2.59448 & 4.61636 & 0.79645 \\
\hline $\mathrm{C}$ & -2.16333 & 3.82506 & -0.37427 & $\mathrm{C}$ & -2.03699 & 3.95232 & -0.28847 \\
\hline $\mathrm{H}$ & -5.34704 & -4.43825 & -0.10355 & $\mathrm{H}$ & -5.34932 & -4.42001 & 0.15623 \\
\hline $\mathrm{H}$ & -2.98121 & -3.79108 & -0.35093 & $\mathrm{H}$ & -2.98801 & -3.77094 & -0.09736 \\
\hline $\mathrm{H}$ & -6.40860 & -0.31970 & 0.41174 & $\mathrm{H}$ & -6.47261 & -0.27789 & 0.15533 \\
\hline $\mathrm{H}$ & -4.05546 & 0.32497 & 0.18008 & $\mathrm{H}$ & -4.12743 & 0.36703 & -0.08990 \\
\hline $\mathrm{H}$ & -1.22978 & -2.18813 & -0.25249 & $\mathrm{H}$ & -1.27267 & -2.17069 & -0.20464 \\
\hline $\mathrm{H}$ & -2.07139 & 0.72418 & -0.60102 & $\mathrm{H}$ & -2.09280 & 0.73368 & -0.67381 \\
\hline $\mathrm{H}$ & 3.08887 & -2.38730 & -0.14355 & $\mathrm{H}$ & 3.05267 & -2.41637 & -0.18727 \\
\hline $\mathrm{H}$ & 5.75875 & -1.74480 & -1.15890 & $\mathrm{H}$ & 5.74747 & -1.76720 & -1.15254 \\
\hline $\mathrm{H}$ & 5.20841 & -3.29552 & -0.51649 & $\mathrm{H}$ & 5.16262 & -3.33239 & -0.58037 \\
\hline $\mathrm{H}$ & 5.95742 & -0.83032 & 1.12059 & $\mathrm{H}$ & 5.83435 & -0.92837 & 1.18540 \\
\hline $\mathrm{H}$ & 5.34525 & -2.35365 & 1.80845 & $\mathrm{H}$ & 5.23548 & -2.49557 & 1.77385 \\
\hline $\mathrm{H}$ & 8.95009 & -2.66790 & 1.88915 & $\mathrm{H}$ & 8.82722 & -2.74961 & 2.00974 \\
\hline $\mathrm{H}$ & 7.51817 & -2.61872 & 2.94642 & $\mathrm{H}$ & 7.33561 & -2.81453 & 2.98040 \\
\hline $\mathrm{H}$ & 8.11589 & -1.12214 & 2.18241 & $\mathrm{H}$ & 7.93117 & -1.25173 & 2.36307 \\
\hline $\mathrm{H}$ & 4.80667 & 2.35757 & -1.53156 & $\mathrm{H}$ & 4.79589 & 2.31765 & -1.60123 \\
\hline $\mathrm{H}$ & -0.93622 & 2.34951 & -2.15234 & $\mathrm{H}$ & -1.01228 & 2.35157 & -2.10423 \\
\hline $\mathrm{H}$ & 0.52491 & 3.24508 & -1.75523 & $\mathrm{H}$ & 0.48074 & 3.23073 & -1.82748 \\
\hline $\mathrm{H}$ & 0.59472 & 2.73857 & 1.25361 & $\mathrm{H}$ & 0.67780 & 2.61802 & 1.22950 \\
\hline $\mathrm{H}$ & -0.55369 & 3.85478 & 3.12537 & $\mathrm{H}$ & -0.31228 & 3.78759 & 3.15448 \\
\hline $\mathrm{H}$ & -2.73855 & 4.96116 & 2.76471 & $\mathrm{H}$ & -2.41229 & 5.07303 & 2.88780 \\
\hline $\mathrm{H}$ & -3.76636 & 4.94100 & 0.51154 & $\mathrm{H}$ & -3.51529 & 5.17337 & 0.67118 \\
\hline $\mathrm{H}$ & -2.61957 & 3.81702 & -1.35892 & $\mathrm{H}$ & -2.52704 & 3.99540 & -1.25526 \\
\hline
\end{tabular}

Table S7: Optimized geometries in vacuum and in solvent medium for compound $3 \mathrm{e}$

\begin{tabular}{|c|c|c|c|c|c|c|c|}
\hline \multicolumn{4}{|c|}{$3 \mathrm{f}$ in Vacuum } & & \multicolumn{2}{|c|}{$3 f$ in $\mathrm{DCM}$} & \\
\hline \multicolumn{8}{|c|}{ CAM-B3LYP/6-311+G(d,p) } \\
\hline $\mathrm{F}$ & -7.66507 & -1.60216 & -1.00147 & $\mathrm{~F}$ & -7.65435 & -1.87457 & -1.02557 \\
\hline $\mathrm{C}$ & -7.05394 & -1.79407 & 0.18698 & $\mathrm{C}$ & -7.06965 & -1.77109 & 0.19080 \\
\hline $\mathrm{C}$ & -5.57442 & -1.57877 & 0.09323 & $\mathrm{C}$ & -5.59540 & -1.53887 & 0.08796 \\
\hline $\mathrm{C}$ & -4.72367 & -2.65157 & -0.12694 & $\mathrm{C}$ & -4.71889 & -2.61420 & 0.10108 \\
\hline $\mathrm{C}$ & -3.36205 & -2.43107 & -0.25402 & $\mathrm{C}$ & -3.35912 & -2.38882 & -0.03871 \\
\hline $\mathrm{C}$ & -5.06007 & -0.28959 & 0.18845 & $\mathrm{C}$ & -5.11153 & -0.24281 & -0.06607 \\
\hline $\mathrm{C}$ & -3.70127 & -0.07691 & 0.05903 & $\mathrm{C}$ & -3.75424 & -0.02619 & -0.20573 \\
\hline $\mathrm{C}$ & -2.82671 & -1.14369 & -0.17617 & $\mathrm{C}$ & -2.85246 & -1.09676 & -0.19930 \\
\hline $\mathrm{C}$ & -1.38098 & -0.96933 & -0.33395 & $\mathrm{C}$ & -1.40458 & -0.92735 & -0.34631 \\
\hline $\mathrm{C}$ & -0.74527 & 0.18731 & -0.53856 & $\mathrm{C}$ & -0.76049 & 0.21453 & -0.60442 \\
\hline $\mathrm{C}$ & 0.69912 & 0.27364 & -0.66724 & $\mathrm{C}$ & 0.68578 & 0.28650 & -0.72561 \\
\hline $\mathrm{N}$ & 1.54633 & -0.71101 & -0.47689 & $\mathrm{~N}$ & 1.52462 & -0.71019 & -0.54731 \\
\hline $\mathrm{C}$ & 2.78037 & -0.16418 & -0.71291 & $\mathrm{C}$ & 2.76489 & -0.17145 & -0.77028 \\
\hline $\mathrm{C}$ & 4.08451 & -0.69476 & -0.67488 & $\mathrm{C}$ & 4.06348 & -0.71851 & -0.73379 \\
\hline $\mathrm{N}$ & 4.30654 & -1.98830 & -0.36379 & $\mathrm{~N}$ & 4.27032 & -2.01680 & -0.44426 \\
\hline $\mathrm{C}$ & 5.61851 & -2.60466 & -0.34365 & $\mathrm{C}$ & 5.57485 & -2.64652 & -0.38941 \\
\hline $\mathrm{C}$ & 6.34091 & -2.38856 & 0.97659 & $\mathrm{C}$ & 6.23232 & -2.48714 & 0.97235 \\
\hline $\mathrm{O}$ & 7.56390 & -3.08165 & 0.90871 & $\mathrm{O}$ & 7.47938 & -3.14524 & 0.92851 \\
\hline $\mathrm{C}$ & 8.36285 & -2.90528 & 2.05278 & $\mathrm{C}$ & 8.19540 & -3.04266 & 2.14146 \\
\hline $\mathrm{N}$ & 5.10887 & 0.11281 & -0.96125 & $\mathrm{~N}$ & 5.09797 & 0.08704 & -1.00427 \\
\hline $\mathrm{C}$ & 4.85445 & 1.38794 & -1.27865 & $\mathrm{C}$ & 4.85625 & 1.36931 & -1.30249 \\
\hline $\mathrm{N}$ & 3.68184 & 1.99962 & -1.34570 & $\mathrm{~N}$ & 3.68968 & 1.99296 & -1.36590 \\
\hline $\mathrm{C}$ & 2.67510 & 1.17509 & -1.05103 & $\mathrm{C}$ & 2.67017 & 1.17364 & -1.08843 \\
\hline $\mathrm{N}$ & 1.33257 & 1.45544 & -1.01910 & $\mathrm{~N}$ & 1.33143 & 1.46479 & -1.05784 \\
\hline $\mathrm{C}$ & 0.74436 & 2.74385 & -1.35059 & $\mathrm{C}$ & 0.74725 & 2.76810 & -1.34207 \\
\hline $\mathrm{C}$ & 0.10783 & 3.45743 & -0.17848 & $\mathrm{C}$ & 0.18668 & 3.47403 & -0.12655 \\
\hline $\mathrm{C}$ & -1.04747 & 4.20730 & -0.37317 & $\mathrm{C}$ & -0.92201 & 4.30309 & -0.26961 \\
\hline
\end{tabular}




\begin{tabular}{|c|c|c|c|c|c|c|c|}
\hline $\mathrm{C}$ & -1.62243 & 4.90740 & 0.67954 & $\mathrm{C}$ & -1.42650 & 5.00252 & 0.81917 \\
\hline $\mathrm{C}$ & -1.04774 & 4.85841 & 1.94133 & $\mathrm{C}$ & -0.82880 & 4.87406 & 2.06592 \\
\hline $\mathrm{C}$ & 0.10445 & 4.10938 & 2.14266 & $\mathrm{C}$ & 0.27514 & 4.04410 & 2.21591 \\
\hline $\mathrm{C}$ & 0.67998 & 3.41439 & 1.08908 & $\mathrm{C}$ & 0.78112 & 3.34927 & 1.12551 \\
\hline $\mathrm{F}$ & -7.63469 & -0.94522 & 1.05441 & $\mathrm{~F}$ & -7.70379 & -0.76761 & 0.82652 \\
\hline $\mathrm{F}$ & -7.36448 & -3.04063 & 0.58118 & $\mathrm{~F}$ & -7.36839 & -2.90512 & 0.84981 \\
\hline $\mathrm{H}$ & -5.12204 & -3.65576 & -0.18958 & $\mathrm{H}$ & -5.09178 & -3.62224 & 0.22667 \\
\hline $\mathrm{H}$ & -2.69848 & -3.27136 & -0.42112 & $\mathrm{H}$ & -2.67587 & -3.22944 & -0.02306 \\
\hline $\mathrm{H}$ & -5.72437 & 0.54335 & 0.38050 & $\mathrm{H}$ & -5.79519 & 0.59636 & -0.06659 \\
\hline $\mathrm{H}$ & -3.31524 & 0.92923 & 0.16293 & $\mathrm{H}$ & -3.39765 & 0.99012 & -0.31106 \\
\hline $\mathrm{H}$ & -0.78038 & -1.87255 & -0.29236 & $\mathrm{H}$ & -0.81161 & -1.82976 & -0.23735 \\
\hline $\mathrm{H}$ & -1.29465 & 1.11766 & -0.61345 & $\mathrm{H}$ & -1.30171 & 1.14340 & -0.73190 \\
\hline $\mathrm{H}$ & 3.49198 & -2.54115 & -0.15015 & $\mathrm{H}$ & 3.45339 & -2.56854 & -0.23379 \\
\hline $\mathrm{H}$ & 6.22051 & -2.19027 & -1.15180 & $\mathrm{H}$ & 6.21479 & -2.20850 & -1.15465 \\
\hline $\mathrm{H}$ & 5.50065 & -3.67467 & -0.52037 & $\mathrm{H}$ & 5.45145 & -3.70638 & -0.61403 \\
\hline $\mathrm{H}$ & 6.50924 & -1.31564 & 1.13359 & $\mathrm{H}$ & 6.36795 & -1.42238 & 1.19950 \\
\hline $\mathrm{H}$ & 5.73070 & -2.76623 & 1.81041 & $\mathrm{H}$ & 5.59682 & -2.92531 & 1.75395 \\
\hline $\mathrm{H}$ & 9.27900 & -3.47455 & 1.90188 & $\mathrm{H}$ & 9.13590 & -3.57612 & 2.01255 \\
\hline $\mathrm{H}$ & 7.85672 & -3.27458 & 2.95440 & $\mathrm{H}$ & 7.63642 & -3.49424 & 2.96970 \\
\hline $\mathrm{H}$ & 8.61790 & -1.84834 & 2.20137 & $\mathrm{H}$ & 8.40657 & -1.99544 & 2.38779 \\
\hline $\mathrm{H}$ & 5.72558 & 1.99343 & -1.50697 & $\mathrm{H}$ & 5.73410 & 1.97065 & -1.51689 \\
\hline $\mathrm{H}$ & 0.01511 & 2.61150 & -2.15249 & $\mathrm{H}$ & -0.02347 & 2.65611 & -2.10564 \\
\hline $\mathrm{H}$ & 1.56512 & 3.34145 & -1.75286 & $\mathrm{H}$ & 1.55020 & 3.36170 & -1.78213 \\
\hline $\mathrm{H}$ & -1.50301 & 4.24689 & -1.35737 & $\mathrm{H}$ & -1.39719 & 4.40226 & -1.23978 \\
\hline $\mathrm{H}$ & -2.52203 & 5.48786 & 0.51313 & $\mathrm{H}$ & -2.29098 & 5.64339 & 0.69338 \\
\hline $\mathrm{H}$ & -1.49596 & 5.39996 & 2.76548 & $\mathrm{H}$ & -1.22320 & 5.41488 & 2.91766 \\
\hline $\mathrm{H}$ & 0.55888 & 4.06724 & 3.12525 & $\mathrm{H}$ & 0.74605 & 3.93713 & 3.18579 \\
\hline $\mathrm{H}$ & 1.58015 & 2.83370 & 1.25328 & $\mathrm{H}$ & 1.64282 & 2.70466 & 1.25391 \\
\hline
\end{tabular}

Table S8: Optimized geometries in vacuum and in solvent medium for compound $3 \mathrm{f}$

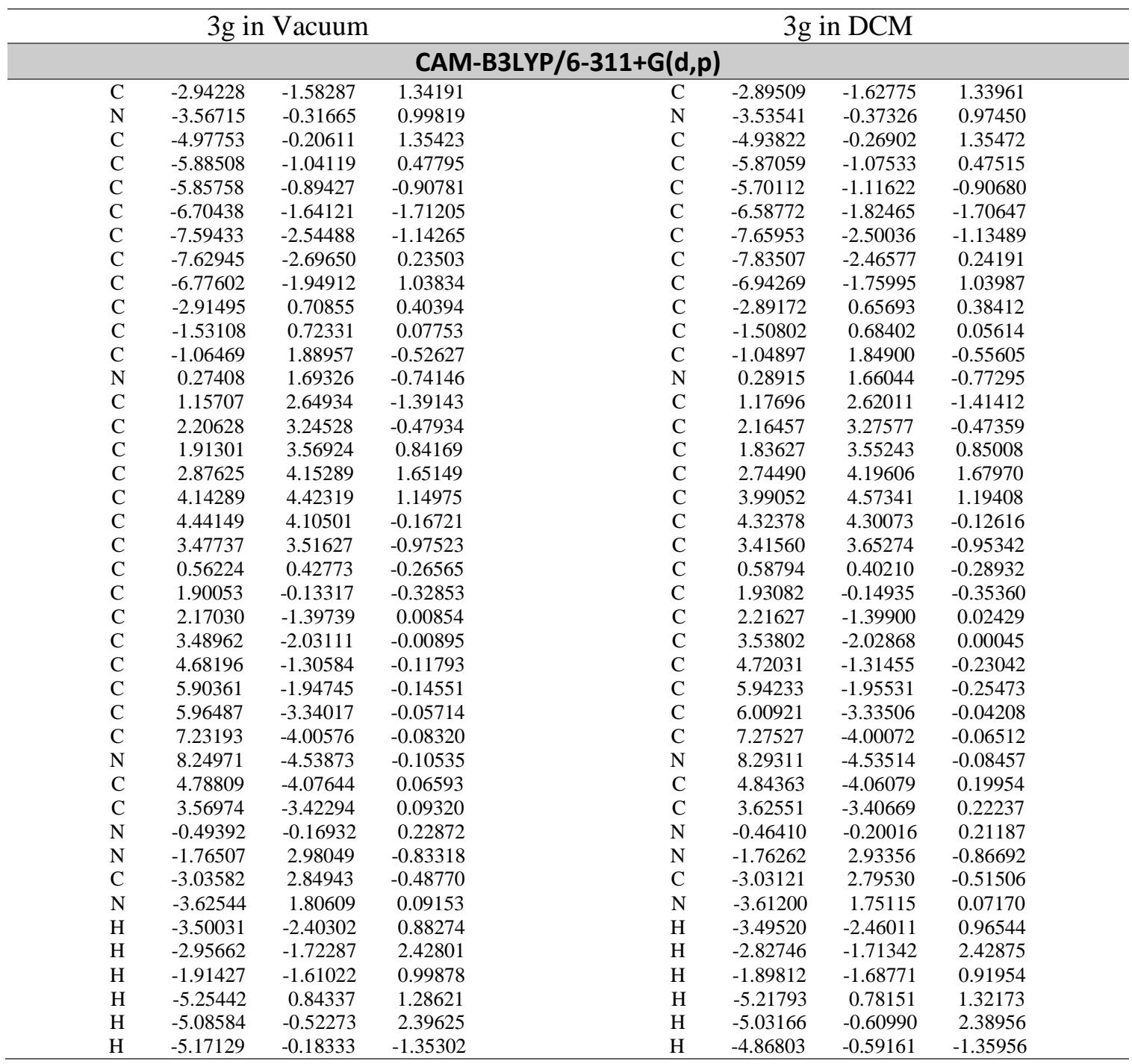




\begin{tabular}{crrrrrrr}
\hline $\mathrm{H}$ & -6.67438 & -1.51563 & -2.78802 & $\mathrm{H}$ & -6.44248 & -1.84806 & -2.78016 \\
$\mathrm{H}$ & -8.25599 & -3.12767 & -1.77224 & $\mathrm{H}$ & -8.35071 & -3.05369 & -1.75934 \\
$\mathrm{H}$ & -8.31755 & -3.40061 & 0.68777 & $\mathrm{H}$ & -8.66343 & -2.99486 & 0.69820 \\
$\mathrm{H}$ & -6.80414 & -2.07516 & 2.11566 & $\mathrm{H}$ & -7.08026 & -1.74416 & 2.11589 \\
$\mathrm{H}$ & 0.50141 & 3.43446 & -1.77338 & $\mathrm{H}$ & 0.52494 & 3.37459 & -1.85683 \\
$\mathrm{H}$ & 1.63180 & 2.17328 & -2.25192 & $\mathrm{H}$ & 1.70232 & 2.12857 & -2.23398 \\
$\mathrm{H}$ & 0.92597 & 3.36387 & 1.23929 & $\mathrm{H}$ & 0.86715 & 3.26314 & 1.23937 \\
$\mathrm{H}$ & 2.63592 & 4.39979 & 2.67863 & $\mathrm{H}$ & 2.47772 & 4.40405 & 2.70889 \\
$\mathrm{H}$ & 4.89378 & 4.87877 & 1.78378 & $\mathrm{H}$ & 4.69896 & 5.07444 & 1.84261 \\
$\mathrm{H}$ & 5.42707 & 4.31141 & -0.56717 & $\mathrm{H}$ & 5.29454 & 4.58709 & -0.51281 \\
$\mathrm{H}$ & 3.71730 & 3.26725 & -2.00391 & $\mathrm{H}$ & 3.68403 & 3.43690 & -1.98212 \\
$\mathrm{H}$ & 2.68725 & 0.53176 & -0.66323 & $\mathrm{H}$ & 2.70483 & 0.50775 & -0.72964 \\
$\mathrm{H}$ & 1.33934 & -2.02601 & 0.31235 & $\mathrm{H}$ & 1.40027 & -2.02408 & 0.37215 \\
$\mathrm{H}$ & 4.65745 & -0.22450 & -0.16396 & $\mathrm{H}$ & 4.69166 & -0.24333 & -0.38123 \\
$\mathrm{H}$ & 6.81970 & -1.37652 & -0.22659 & $\mathrm{H}$ & 6.84956 & -1.39238 & -0.43185 \\
$\mathrm{H}$ & 4.83446 & -5.15522 & 0.14088 & $\mathrm{H}$ & 4.89478 & -5.12839 & 0.36888 \\
$\mathrm{H}$ & 2.65752 & -3.99985 & 0.18937 & $\mathrm{H}$ & 2.72159 & -3.97310 & 0.41125 \\
\hline
\end{tabular}

Table S9: Optimized geometries in vacuum and in solvent medium for compound $3 \mathrm{~g}$

\begin{tabular}{|c|c|c|c|c|c|c|c|}
\hline \multicolumn{4}{|c|}{ 7a in Vacuum } & \multicolumn{4}{|c|}{$7 \mathrm{a}$ in DMSO } \\
\hline \multicolumn{8}{|c|}{ CAM-B3LYP/6-311+G(d,p) } \\
\hline $\mathrm{N}$ & -8.98065 & -3.40451 & -0.01916 & $\mathrm{~N}$ & -8.97022 & -3.35219 & 0.01340 \\
\hline $\mathrm{C}$ & -8.00185 & -2.80268 & -0.02361 & $\mathrm{C}$ & -7.98640 & -2.75700 & 0.00890 \\
\hline $\mathrm{C}$ & -6.78369 & -2.05087 & -0.02891 & $\mathrm{C}$ & -6.76316 & -2.01550 & 0.00316 \\
\hline $\mathrm{C}$ & -6.82176 & -0.65563 & 0.03274 & $\mathrm{C}$ & -6.79514 & -0.61800 & 0.00586 \\
\hline $\mathrm{C}$ & -5.64748 & 0.06865 & 0.02686 & $\mathrm{C}$ & -5.61454 & 0.09617 & -0.00010 \\
\hline $\mathrm{C}$ & -5.55558 & -2.70503 & -0.09720 & $\mathrm{C}$ & -5.54101 & -2.68603 & -0.00552 \\
\hline $\mathrm{C}$ & -4.38523 & -1.96818 & -0.10255 & $\mathrm{C}$ & -4.36498 & -1.95832 & -0.01144 \\
\hline $\mathrm{C}$ & -4.40447 & -0.57195 & -0.03892 & $\mathrm{C}$ & -4.37720 & -0.55988 & -0.00859 \\
\hline $\mathrm{C}$ & -3.12906 & 0.14526 & -0.04569 & $\mathrm{C}$ & -3.09485 & 0.14720 & -0.01509 \\
\hline $\mathrm{C}$ & -2.95144 & 1.46526 & 0.05404 & $\mathrm{C}$ & -2.91676 & 1.47123 & -0.00566 \\
\hline $\mathrm{C}$ & -1.63719 & 2.08172 & 0.03708 & $\mathrm{C}$ & -1.60173 & 2.08775 & -0.01352 \\
\hline $\mathrm{N}$ & -0.48778 & 1.46438 & -0.08403 & $\mathrm{~N}$ & -0.44193 & 1.47026 & -0.03263 \\
\hline $\mathrm{C}$ & 0.45471 & 2.46166 & -0.04405 & $\mathrm{C}$ & 0.49216 & 2.47634 & -0.03302 \\
\hline $\mathrm{C}$ & 1.86268 & 2.44812 & -0.12447 & $\mathrm{C}$ & 1.90312 & 2.46589 & -0.04990 \\
\hline $\mathrm{N}$ & 2.54925 & 1.29827 & -0.25453 & $\mathrm{~N}$ & 2.60183 & 1.32056 & -0.06171 \\
\hline $\mathrm{C}$ & 3.99665 & 1.23509 & -0.40561 & $\mathrm{C}$ & 4.05924 & 1.25885 & -0.11994 \\
\hline $\mathrm{C}$ & 4.49718 & -0.16203 & -0.15271 & $\mathrm{C}$ & 4.52950 & -0.16447 & 0.01475 \\
\hline $\mathrm{C}$ & 4.42733 & -0.72342 & 1.12609 & $\mathrm{C}$ & 4.63334 & -0.76609 & 1.27146 \\
\hline $\mathrm{C}$ & 4.87994 & -2.00279 & 1.36910 & $\mathrm{C}$ & 5.04209 & -2.07879 & 1.40205 \\
\hline $\mathrm{C}$ & 5.42195 & -2.76279 & 0.33039 & $\mathrm{C}$ & 5.36020 & -2.82871 & 0.26793 \\
\hline $\mathrm{O}$ & 5.84253 & -4.00920 & 0.66656 & $\mathrm{O}$ & 5.75384 & -4.10926 & 0.49232 \\
\hline $\mathrm{C}$ & 6.40986 & -4.82312 & -0.33978 & $\mathrm{C}$ & 6.08971 & -4.91974 & -0.62539 \\
\hline $\mathrm{C}$ & 5.50128 & -2.22274 & -0.94623 & $\mathrm{C}$ & 5.26586 & -2.24636 & -0.99107 \\
\hline $\mathrm{C}$ & 5.03534 & -0.92907 & -1.17265 & $\mathrm{C}$ & 4.85073 & -0.92180 & -1.10244 \\
\hline $\mathrm{N}$ & 2.51298 & 3.61328 & -0.05967 & $\mathrm{~N}$ & 2.54506 & 3.64250 & -0.04935 \\
\hline $\mathrm{C}$ & 1.80496 & 4.73984 & 0.08820 & $\mathrm{C}$ & 1.82402 & 4.77073 & -0.02737 \\
\hline $\mathrm{N}$ & 0.49155 & 4.88145 & 0.17684 & $\mathrm{~N}$ & 0.50723 & 4.90642 & -0.00919 \\
\hline $\mathrm{C}$ & -0.13622 & 3.70726 & 0.10364 & $\mathrm{C}$ & -0.11636 & 3.72223 & -0.01374 \\
\hline $\mathrm{N}$ & -1.48024 & 3.44911 & 0.15458 & $\mathrm{~N}$ & -1.45653 & 3.45718 & -0.00116 \\
\hline $\mathrm{H}$ & -7.77702 & -0.14911 & 0.08337 & $\mathrm{H}$ & -7.74513 & -0.09972 & 0.01231 \\
\hline $\mathrm{H}$ & -5.70276 & 1.14871 & 0.07123 & $\mathrm{H}$ & -5.66284 & 1.17703 & 0.00147 \\
\hline $\mathrm{H}$ & -5.52402 & -3.78580 & -0.14595 & $\mathrm{H}$ & -5.51485 & -3.76784 & -0.00771 \\
\hline $\mathrm{H}$ & -3.43263 & -2.48177 & -0.15575 & $\mathrm{H}$ & -3.41653 & -2.48158 & -0.01824 \\
\hline $\mathrm{H}$ & -2.23805 & -0.46783 & -0.13827 & $\mathrm{H}$ & -2.21143 & -0.48305 & -0.02845 \\
\hline $\mathrm{H}$ & -3.79430 & 2.14068 & 0.15611 & $\mathrm{H}$ & -3.75681 & 2.15603 & 0.00879 \\
\hline $\mathrm{H}$ & 2.01290 & 0.45141 & -0.36425 & $\mathrm{H}$ & 2.08182 & 0.45764 & -0.10750 \\
\hline $\mathrm{H}$ & 4.43697 & 1.94440 & 0.29702 & $\mathrm{H}$ & 4.46755 & 1.87593 & 0.68284 \\
\hline $\mathrm{H}$ & 4.28986 & 1.56456 & -1.40738 & $\mathrm{H}$ & 4.40941 & 1.68612 & -1.06329 \\
\hline $\mathrm{H}$ & 4.01212 & -0.14140 & 1.94169 & $\mathrm{H}$ & 4.39390 & -0.19373 & 2.16107 \\
\hline $\mathrm{H}$ & 4.83209 & -2.44029 & 2.35830 & $\mathrm{H}$ & 5.12694 & -2.54395 & 2.37643 \\
\hline $\mathrm{H}$ & 6.67489 & -5.75987 & 0.14516 & $\mathrm{H}$ & 6.36858 & -5.88901 & -0.21975 \\
\hline $\mathrm{H}$ & 7.31088 & -4.36649 & -0.76067 & $\mathrm{H}$ & 6.93451 & -4.50024 & -1.17729 \\
\hline $\mathrm{H}$ & 5.69341 & -5.02205 & -1.14271 & $\mathrm{H}$ & 5.23584 & -5.03884 & -1.29697 \\
\hline $\mathrm{H}$ & 5.91725 & -2.78758 & -1.76848 & $\mathrm{H}$ & 5.51077 & -2.80211 & -1.88497 \\
\hline $\mathrm{H}$ & 5.10144 & -0.51636 & -2.17348 & $\mathrm{H}$ & 4.78252 & -0.47653 & -2.08897 \\
\hline $\mathrm{H}$ & 2.38953 & 5.65274 & 0.13848 & $\mathrm{H}$ & 2.40042 & 5.69060 & -0.02594 \\
\hline $\mathrm{H}$ & -2.20595 & 4.14028 & 0.26033 & $\mathrm{H}$ & -2.20044 & 4.13999 & 0.01408 \\
\hline
\end{tabular}

Table S10: Optimized geometries in vacuum and in solvent medium for compound $7 \mathrm{a}$ 


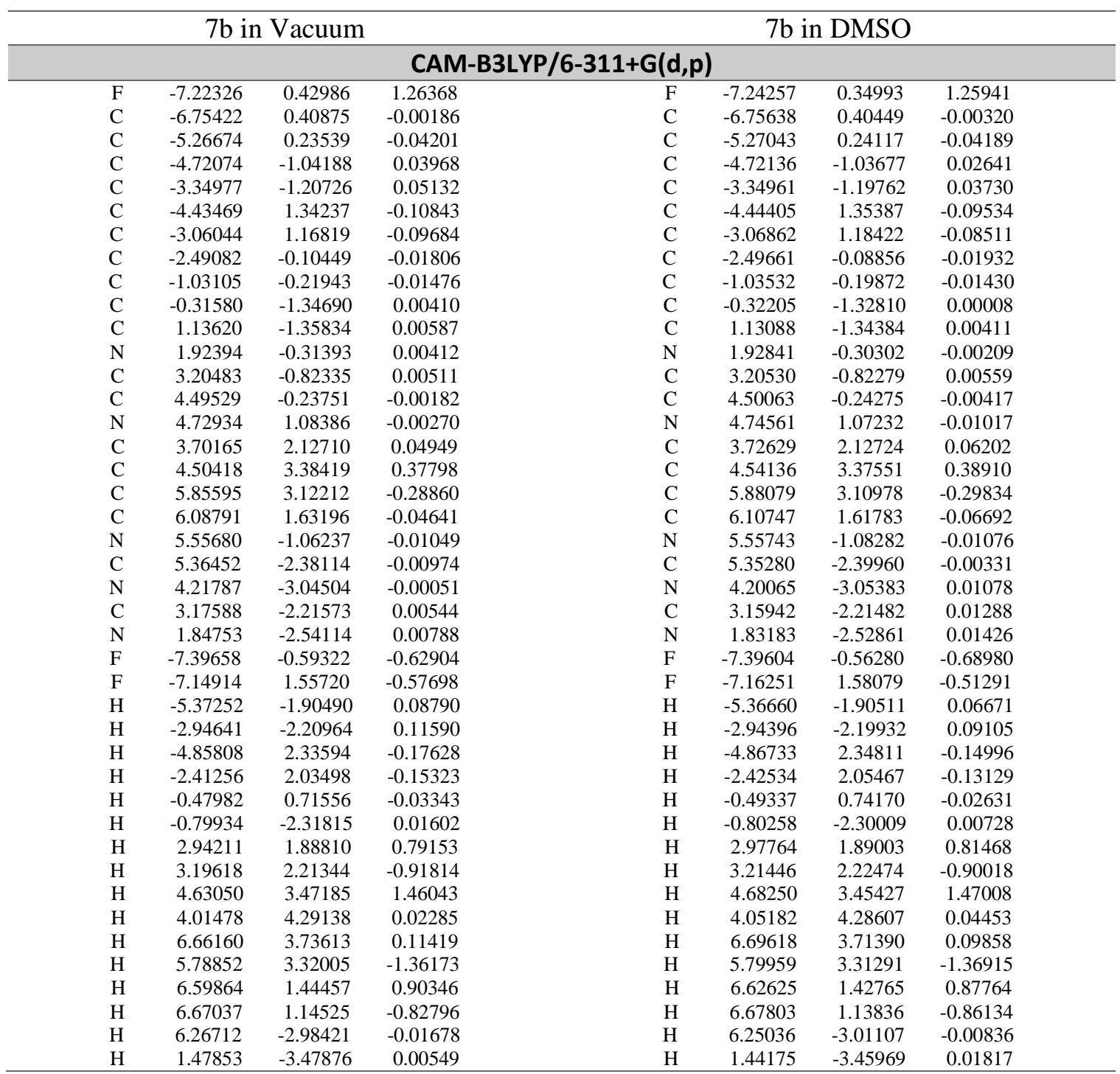

Table S11: Optimized geometries in vacuum and in solvent medium for compound $7 \mathrm{~b}$

c) Excited states, transition energy, and oscillator strength

\section{Compound 3a}

TDA-CAM-B3LYP/6-311+G(d,p)

\begin{tabular}{cccccccc}
\hline \multicolumn{1}{c}{ Vacuum } & \multicolumn{5}{c}{ DCM } \\
$\begin{array}{c}\text { excited } \\
\text { state }\end{array}$ & $\mathrm{eV}$ & $\mathrm{nm}$ & $\mathrm{f}$ & $\begin{array}{c}\text { excited } \\
\text { state }\end{array}$ & $\mathrm{eV}$ & $\mathrm{nm}$ & $\mathrm{f}$ \\
\hline 1 & 3.7675 & 329.09 & 1.2162 & 1 & 3.5395 & 350.29 & 1.4979 \\
\hline 2 & 4.8505 & 255.61 & 0.0692 & 2 & 4.8082 & 257.86 & 0.2697 \\
\hline 3 & 4.8981 & 253.13 & 0.1158 & 3 & 4.8558 & 255.33 & 0.1591 \\
\hline 4 & 4.9663 & 249.65 & 0.1093 & 4 & 5.0269 & 246.64 & 0.0067 \\
\hline 5 & 5.2081 & 238.06 & 0.0313 & 5 & 5.1682 & 239.90 & 0.0833 \\
\hline 6 & 5.2556 & 235.91 & 0.1061 & 6 & 5.1851 & 239.12 & 0.0464 \\
\hline 7 & 5.3343 & 232.43 & 0.0141 & 7 & 5.3144 & 233.30 & 0.0628 \\
\hline 8 & 5.4254 & 228.52 & 0.1892 & 8 & 5.3353 & 232.38 & 0.1282 \\
\hline 9 & 5.4565 & 227.22 & 0.0500 & 9 & 5.4015 & 229.54 & 0.1162 \\
\hline 10 & 5.5226 & 224.50 & 0.0174 & 10 & 5.4908 & 225.80 & 0.0045 \\
\hline 11 & 5.5309 & 224.17 & 0.0230 & 11 & 5.5738 & 222.44 & 0.0007 \\
\hline
\end{tabular}




\begin{tabular}{llllllll|}
\hline 12 & 5.6208 & 220.58 & 0.0106 & 12 & 5.6186 & 220.67 & 0.0324 \\
\hline 13 & 5.7226 & 216.66 & 0.0582 & 13 & 5.6774 & 218.38 & 0.0763 \\
\hline 14 & 5.7815 & 214.45 & 0.0008 & 14 & 5.6886 & 217.95 & 0.1246 \\
\hline 15 & 5.7964 & 213.90 & 0.0026 & 15 & 5.8191 & 213.06 & 0.4968 \\
\hline
\end{tabular}

Table S12:Excited states, transition energy, and oscillator strength for compound 3a in gas-phase and solvent medium.

\section{Compound 3b \\ TDA-CAM-B3LYP/6-311+G(d,p)}

\begin{tabular}{cccccccc}
\hline \multicolumn{1}{c}{ Vacuum } & \multicolumn{5}{c}{ DCM } \\
$\begin{array}{c}\text { excited } \\
\text { state }\end{array}$ & $\mathrm{eV}$ & $\mathrm{nm}$ & $\mathrm{f}$ & $\begin{array}{c}\text { excited } \\
\text { state }\end{array}$ & $\mathrm{eV}$ & $\mathrm{nm}$ & $\mathrm{f}$ \\
\hline 1 & 3.8668 & 320.64 & 1.1040 & 1 & 3.6686 & 337.96 & 1.3455 \\
\hline 2 & 4.9328 & 251.35 & 0.0494 & 2 & 4.9147 & 252.27 & 0.1468 \\
\hline 3 & 4.9737 & 249.28 & 0.0361 & 3 & 4.9823 & 248.85 & 0.2189 \\
\hline 4 & 5.0377 & 246.11 & 0.1289 & 4 & 5.0920 & 243.49 & 0.0094 \\
\hline 5 & 5.2210 & 237.47 & 0.0332 & 5 & 5.1965 & 238.59 & 0.0582 \\
\hline 6 & 5.3464 & 231.90 & 0.1590 & 6 & 5.2605 & 235.69 & 0.1917 \\
\hline 7 & 5.4472 & 227.61 & 0.0067 & 7 & 5.4235 & 228.61 & 0.0744 \\
\hline 8 & 5.4789 & 226.29 & 0.0251 & 8 & 5.4477 & 227.59 & 0.1028 \\
\hline 9 & 5.5098 & 225.03 & 0.0317 & 9 & 5.4816 & 226.18 & 0.0983 \\
\hline 10 & 5.5353 & 223.99 & 0.2039 & 10 & 5.5302 & 224.19 & 0.0444 \\
\hline 11 & 5.5870 & 221.92 & 0.0583 & 11 & 5.5614 & 222.94 & 0.0019 \\
\hline 12 & 5.6819 & 218.21 & 0.0171 & 12 & 5.7169 & 216.87 & 0.0472 \\
\hline 13 & 5.7769 & 214.62 & 0.0076 & 13 & 5.7740 & 214.73 & 0.1335 \\
\hline 14 & 5.8053 & 213.57 & 0.0546 & 14 & 5.7888 & 214.18 & 0.2847 \\
\hline 15 & 5.8762 & 211.00 & 0.0947 & 15 & 5.9340 & 208.94 & 0.1988 \\
\hline
\end{tabular}

Table S13: Excited states, transition energy, and oscillator strength for compound $3 \mathrm{~b}$ in gas-phase and solvent medium.

\section{Compound 3c}

TDA-CAM-B3LYP/6-311+G(d,p)

\begin{tabular}{cccccccc}
\hline \multicolumn{1}{c}{ Vacuum } & \multicolumn{5}{c}{ DCM } \\
\hline $\begin{array}{c}\text { excited } \\
\text { state }\end{array}$ & $\mathrm{eV}$ & $\mathrm{nm}$ & $\mathrm{f}$ & $\begin{array}{c}\text { excited } \\
\text { state }\end{array}$ & $\mathrm{eV}$ & $\mathrm{nm}$ & $\mathrm{f}$ \\
\hline 1 & 3.6778 & 337.12 & 1.1362 & 1 & 3.4822 & 356.06 & 1.4193 \\
\hline 2 & 4.7838 & 259.17 & 0.2640 & 2 & 4.7270 & 262.29 & 0.4301 \\
\hline 3 & 4.8562 & 255.31 & 0.0937 & 3 & 4.8399 & 256.17 & 0.0290 \\
\hline 4 & 4.9638 & 249.78 & 0.0003 & 4 & 5.0824 & 243.95 & 0.0060 \\
\hline 5 & 5.2140 & 237.79 & 0.1510 & 5 & 5.1349 & 241.45 & 0.1652 \\
\hline 6 & 5.3128 & 233.37 & 0.0391 & 6 & 5.2560 & 235.89 & 0.2582 \\
\hline 7 & 5.3924 & 229.92 & 0.1992 & 7 & 5.3443 & 231.99 & 0.0454 \\
\hline 8 & 5.4018 & 229.52 & 0.0442 & 8 & 5.3824 & 230.35 & 0.0187 \\
\hline 9 & 5.4908 & 225.80 & 0.0017 & 9 & 5.4892 & 225.87 & 0.0060 \\
\hline 10 & 5.5727 & 222.48 & 0.0104 & 10 & 5.5984 & 221.46 & 0.0873 \\
\hline 11 & 5.6159 & 220.77 & 0.0192 & 11 & 5.6083 & 221.07 & 0.0380 \\
\hline 12 & 5.6642 & 218.89 & 0.0028 & 12 & 5.6773 & 218.39 & 0.0353 \\
\hline 13 & 5.7306 & 216.36 & 0.0547 & 13 & 5.7503 & 215.61 & 0.4129 \\
\hline 14 & 5.7865 & 214.26 & 0.0127 & 14 & 5.8100 & 213.40 & 0.0473 \\
\hline 15 & 5.8564 & 211.71 & 0.0604 & 15 & 5.8384 & 212.36 & 0.0057 \\
\hline
\end{tabular}

Table S14: Excited states, transition energy, and oscillator strength for compound 3c in gas-phase and solvent medium. 


\section{Compound 3d}

TDA-CAM-B3LYP/6-311+G(d,p)

\begin{tabular}{cccccccc}
\hline $\begin{array}{c}\text { excited } \\
\text { state }\end{array}$ & $\mathrm{eV}$ & $\mathrm{nm}$ & $\mathrm{f}$ & $\begin{array}{c}\text { Vexcited } \\
\text { state }\end{array}$ & $\mathrm{eV}$ & $\mathrm{nm}$ & $\mathrm{f}$ \\
\hline 1 & 3.7991 & 326.35 & 1.0294 & 1 & 3.6131 & 343.16 & 1.2814 \\
\hline 2 & 4.8916 & 253.47 & 0.1583 & 2 & 4.8547 & 255.39 & 0.3220 \\
\hline 3 & 4.9550 & 250.22 & 0.0938 & 3 & 4.9425 & 250.85 & 0.0428 \\
\hline 4 & 5.0203 & 246.96 & 0.0005 & 4 & 5.1400 & 241.22 & 0.0046 \\
\hline 5 & 5.2839 & 234.64 & 0.1654 & 5 & 5.1904 & 238.87 & 0.3003 \\
\hline 6 & 5.3191 & 233.09 & 0.0730 & 6 & 5.3439 & 232.01 & 0.2007 \\
\hline 7 & 5.4475 & 227.60 & 0.0145 & 7 & 5.3750 & 230.67 & 0.0819 \\
\hline 8 & 5.4691 & 226.70 & 0.2594 & 8 & 5.4218 & 228.68 & 0.0214 \\
\hline 9 & 5.5137 & 224.86 & 0.0064 & 9 & 5.5150 & 224.81 & 0.0149 \\
\hline 10 & 5.6304 & 220.20 & 0.0066 & 10 & 5.6658 & 218.83 & 0.0320 \\
\hline 11 & 5.6726 & 218.57 & 0.0084 & 11 & 5.7162 & 216.90 & 0.2833 \\
\hline 12 & 5.7016 & 217.46 & 0.0174 & 12 & 5.8038 & 213.63 & 0.0017 \\
\hline 13 & 5.7740 & 214.73 & 0.0870 & 13 & 5.8208 & 213.00 & 0.0235 \\
\hline 14 & 5.8624 & 211.49 & 0.0059 & 14 & 5.8732 & 211.10 & 0.1423 \\
\hline 15 & 5.8970 & 210.25 & 0.0294 & 15 & 5.9255 & 209.24 & 0.0895 \\
\hline
\end{tabular}

Table S15: Excited states, transition energy, and oscillator strength for compound $3 \mathrm{~d}$ in gas-phase and solvent medium.

\section{Compound 3e}

TDA-CAM-B3LYP/6-311+G(d,p)

\begin{tabular}{cccccccc}
\hline $\begin{array}{c}\text { excited } \\
\text { state }\end{array}$ & $\mathrm{eV}$ & $\mathrm{nm}$ & $\mathrm{f}$ & $\begin{array}{c}\text { excited } \\
\text { state }\end{array}$ & $\mathrm{eV}$ & $\mathrm{nm}$ & $\mathrm{f}$ \\
\hline 1 & 3.7875 & 327.35 & 1.2653 & 1 & 3.5701 & 347.28 & 1.5201 \\
\hline 2 & 4.8511 & 255.58 & 0.0315 & 2 & 4.8246 & 256.98 & 0.1603 \\
\hline 3 & 4.9301 & 251.48 & 0.2368 & 3 & 4.8719 & 254.49 & 0.2000 \\
\hline 4 & 4.9884 & 248.54 & 0.0017 & 4 & 5.1068 & 242.78 & 0.0015 \\
\hline 5 & 5.2529 & 236.03 & 0.0878 & 5 & 5.1746 & 239.60 & 0.0507 \\
\hline 6 & 5.4457 & 227.67 & 0.0305 & 6 & 5.3628 & 231.19 & 0.0699 \\
\hline 7 & 5.4676 & 226.76 & 0.1009 & 7 & 5.4177 & 228.85 & 0.1547 \\
\hline 8 & 5.5210 & 224.57 & 0.0203 & 8 & 5.4921 & 225.75 & 0.0054 \\
\hline 9 & 5.5465 & 223.53 & 0.0432 & 9 & 5.5988 & 221.45 & 0.0030 \\
\hline 10 & 5.6316 & 220.16 & 0.0058 & 10 & 5.6266 & 220.35 & 0.0174 \\
\hline 11 & 5.7481 & 215.70 & 0.0906 & 11 & 5.6924 & 217.81 & 0.0022 \\
\hline 12 & 5.7704 & 214.86 & 0.0119 & 12 & 5.7019 & 217.44 & 0.2570 \\
\hline 13 & 5.8298 & 212.67 & 0.0039 & 13 & 5.8352 & 212.48 & 0.2489 \\
\hline 14 & 5.8729 & 211.11 & 0.0452 & 14 & 5.9581 & 208.09 & 0.1800 \\
\hline 15 & 5.9367 & 208.84 & 0.0449 & 15 & 6.0122 & 206.22 & 0.0151 \\
\hline
\end{tabular}

Table S16: Excited states, transition energy, and oscillator strength for compound 3e in gas-phase and solvent medium.

\begin{tabular}{|c|c|c|c|c|c|c|c|}
\hline \multicolumn{8}{|c|}{$\begin{array}{c}\text { Compound 3f } \\
\text { TDA-CAM-B3LYP/6-311+G(d,p) }\end{array}$} \\
\hline \multicolumn{4}{|c|}{ Vacuum } & \multicolumn{4}{|c|}{ DCM } \\
\hline $\begin{array}{l}\text { excited } \\
\text { state }\end{array}$ & $\mathrm{eV}$ & $\mathrm{nm}$ & $\mathrm{f}$ & $\begin{array}{l}\text { excited } \\
\text { state }\end{array}$ & $\mathrm{eV}$ & $\mathrm{nm}$ & $\mathrm{f}$ \\
\hline 1 & 3.9035 & 317.62 & 1.1410 & 1 & 3.6866 & 336.31 & 1.3690 \\
\hline
\end{tabular}




\begin{tabular}{cccccccc}
\hline 2 & 4.9453 & 250.71 & 0.0228 & 2 & 4.9206 & 251.97 & 0.0924 \\
\hline 3 & 5.0295 & 246.51 & 0.1087 & 3 & 4.9932 & 248.30 & 0.1949 \\
\hline 4 & 5.0432 & 245.84 & 0.0587 & 4 & 5.1545 & 240.54 & 0.0013 \\
\hline 5 & 5.3505 & 231.72 & 0.1285 & 5 & 5.2622 & 235.61 & 0.1314 \\
\hline 6 & 5.4709 & 226.62 & 0.0247 & 6 & 5.4358 & 228.09 & 0.0431 \\
\hline 7 & 5.4993 & 225.46 & 0.0172 & 7 & 5.5079 & 225.10 & 0.0969 \\
\hline 8 & 5.5692 & 222.62 & 0.0335 & 8 & 5.5232 & 224.48 & 0.1004 \\
\hline 9 & 5.6135 & 220.87 & 0.1457 & 9 & 5.5855 & 221.97 & 0.0106 \\
\hline 10 & 5.6969 & 217.64 & 0.0130 & 10 & 5.7232 & 216.64 & 0.0408 \\
\hline 11 & 5.8155 & 213.20 & 0.0259 & 11 & 5.7694 & 214.90 & 0.0225 \\
\hline 12 & 5.8401 & 212.30 & 0.0683 & 12 & 5.7979 & 213.84 & 0.3834 \\
\hline 13 & 5.8943 & 210.34 & 0.0454 & 13 & 5.9464 & 208.50 & 0.0699 \\
\hline 14 & 5.9010 & 210.11 & 0.0281 & 14 & 6.0004 & 206.63 & 0.0366 \\
\hline 15 & 5.9968 & 206.75 & 0.0021 & 15 & 6.0482 & 204.99 & 0.0814 \\
\hline Table S17: & Excited states, transition energy, and oscillator strength for compound 3f in gas-phase and solvent \\
medium. & & & & & & \\
\end{tabular}

\section{Compound 3g}

TDA-CAM-B3LYP/6-311+G(d,p)

\begin{tabular}{|c|c|c|c|c|c|c|c|}
\hline \multicolumn{4}{|c|}{ Vacuum } & \multicolumn{4}{|c|}{ DCM } \\
\hline $\begin{array}{l}\text { excited } \\
\text { state }\end{array}$ & $\mathrm{eV}$ & $\mathrm{nm}$ & $f$ & $\begin{array}{c}\text { excited } \\
\text { state }\end{array}$ & $\mathrm{eV}$ & $\mathrm{nm}$ & $f$ \\
\hline 1 & 3.7083 & 334.34 & 1.2022 & 1 & 3.5283 & 351.40 & 1.4685 \\
\hline 2 & 4.7976 & 258.43 & 0.2664 & 2 & 4.7455 & 261.26 & 0.4326 \\
\hline 3 & 4.8685 & 254.67 & 0.0899 & 3 & 4.8460 & 255.85 & 0.0275 \\
\hline 4 & 4.9496 & 250.49 & 0.0001 & 4 & 5.0494 & 245.54 & 0.0022 \\
\hline 5 & 5.2071 & 238.11 & 0.1473 & 5 & 5.1369 & 241.36 & 0.1490 \\
\hline 6 & 5.3711 & 230.83 & 0.1289 & 6 & 5.2992 & 233.97 & 0.2285 \\
\hline 7 & 5.3993 & 229.63 & 0.1717 & 7 & 5.3400 & 232.18 & 0.1078 \\
\hline 8 & 5.4651 & 226.87 & 0.0066 & 8 & 5.4660 & 226.83 & 0.0016 \\
\hline 9 & 5.4827 & 226.14 & 0.0028 & 9 & 5.4878 & 225.93 & 0.0028 \\
\hline 10 & 5.4851 & 226.04 & 0.0089 & 10 & 5.5191 & 224.65 & 0.0080 \\
\hline 11 & 5.5579 & 223.08 & 0.0066 & 11 & 5.5671 & 222.71 & 0.0248 \\
\hline 12 & 5.6757 & 218.45 & 0.0431 & 12 & 5.6494 & 219.46 & 0.1267 \\
\hline 13 & 5.7276 & 216.47 & 0.0080 & 13 & 5.6528 & 219.33 & 0.0080 \\
\hline 14 & 5.7684 & 214.94 & 0.0025 & 14 & 5.7433 & 215.88 & 0.2992 \\
\hline 15 & 5.7728 & 214.77 & 0.0273 & 15 & 5.8144 & 213.24 & 0.0222 \\
\hline
\end{tabular}

Table S18: Excited states, transition energy, and oscillator strength for compound $3 \mathrm{~g}$ in gas-phase and solvent medium.

\section{Compound 7a}

TDA-CAM-B3LYP/6-311+G(d,p)

\begin{tabular}{cccccccc}
\hline $\begin{array}{c}\text { excited } \\
\text { state }\end{array}$ & $\mathrm{eV}$ & $\mathrm{nm}$ & $\mathrm{f}$ & $\begin{array}{c}\text { Vexcited } \\
\text { state }\end{array}$ & $\mathrm{eV}$ & $\mathrm{nm}$ & $\mathrm{f}$ \\
\hline 1 & 3.7524 & 330.41 & 1.2895 & 1 & 3.5675 & 347.54 & 1.5735 \\
\hline 2 & 4.8332 & 256.53 & 0.0506 & 2 & 4.8230 & 257.07 & 0.1581 \\
\hline 3 & 4.8708 & 254.55 & 0.0419 & 3 & 4.8818 & 253.97 & 0.2779 \\
\hline 4 & 4.9670 & 249.61 & 0.2794 & 4 & 5.0699 & 244.55 & 0.0128 \\
\hline 5 & 5.1852 & 239.11 & 0.0178 & 5 & 5.1927 & 238.77 & 0.0601 \\
\hline 6 & 5.2760 & 235.00 & 0.0195 & 6 & 5.2842 & 234.63 & 0.1469 \\
\hline 7 & 5.3786 & 230.51 & 0.1164 & 7 & 5.3619 & 231.23 & 0.0338 \\
\hline
\end{tabular}




\begin{tabular}{cccccccc}
\hline 8 & 5.4193 & 228.78 & 0.0014 & 8 & 5.3912 & 229.98 & 0.1641 \\
\hline 9 & 5.4727 & 226.55 & 0.1957 & 9 & 5.5670 & 222.71 & 0.0004 \\
\hline 10 & 5.5822 & 222.11 & 0.0067 & 10 & 5.7009 & 217.48 & 0.2682 \\
\hline 11 & 5.7596 & 215.27 & 0.1165 & 11 & 5.7332 & 216.26 & 0.0058 \\
\hline 12 & 5.7718 & 214.81 & 0.0017 & 12 & 5.8246 & 212.86 & 0.7614 \\
\hline 13 & 5.9337 & 208.95 & 0.0803 & 13 & 5.9385 & 208.78 & 0.1333 \\
\hline 14 & 5.9673 & 207.77 & 0.0199 & 14 & 6.0520 & 204.86 & 0.1216 \\
\hline 15 & 5.9834 & 207.21 & 0.0590 & 15 & 6.0852 & 203.75 & 0.0100 \\
\hline
\end{tabular}

Table S19: Excited states, transition energy, and oscillator strength for compound 7a in gas-phase and solvent medium.

\begin{tabular}{cccccccc}
\hline \multicolumn{7}{c}{ TDA-CAM-B3LYP/6-311+G(d,p) } \\
\hline \multicolumn{7}{c}{ Vacuum } \\
\hline $\begin{array}{c}\text { excited } \\
\text { state }\end{array}$ & $\mathrm{eV}$ & $\mathrm{nm}$ & $\mathrm{f}$ & $\begin{array}{c}\text { Compound 7b } \\
\text { excited } \\
\text { state }\end{array}$ & $\mathrm{eV}$ & $\mathrm{nm}$ & $\mathrm{f}$ \\
\hline 1 & 3.7921 & 326.95 & 1.0852 & 1 & 3.6378 & 340.82 & 1.3512 \\
\hline 2 & 4.9020 & 252.93 & 0.0714 & 2 & 4.8896 & 253.57 & 0.2797 \\
\hline 3 & 4.9757 & 249.18 & 0.0719 & 3 & 4.9624 & 249.84 & 0.1239 \\
\hline 4 & 4.9914 & 248.40 & 0.1811 & 4 & 5.1867 & 239.04 & 0.0039 \\
\hline 5 & 5.2740 & 235.08 & 0.0087 & 5 & 5.1995 & 238.46 & 0.3046 \\
\hline 6 & 5.3302 & 232.60 & 0.2265 & 6 & 5.3580 & 231.40 & 0.1879 \\
\hline 7 & 5.5046 & 225.24 & 0.1775 & 7 & 5.3774 & 230.57 & 0.0603 \\
\hline 8 & 5.5551 & 223.19 & 0.0060 & 8 & 5.7028 & 217.41 & 0.0068 \\
\hline 9 & 5.5688 & 222.64 & 0.0118 & 9 & 5.7267 & 216.50 & 0.4003 \\
\hline 10 & 5.7674 & 214.97 & 0.1472 & 10 & 5.8889 & 210.54 & 0.2810 \\
\hline 11 & 5.9383 & 208.79 & 0.0031 & 11 & 5.9479 & 208.45 & 0.0062 \\
\hline 12 & 5.9465 & 208.50 & 0.0015 & 12 & 6.0580 & 204.66 & 0.0047 \\
\hline 13 & 5.9684 & 207.73 & 0.1261 & 13 & 6.0612 & 204.55 & 0.0062 \\
\hline 14 & 6.0239 & 205.82 & 0.0065 & 14 & 6.0662 & 204.39 & 0.0044 \\
\hline 15 & 6.1622 & 201.20 & 0.0045 & 15 & 6.2142 & 199.52 & 0.0258 \\
\hline
\end{tabular}

Table S20: Excited states, transition energy, and oscillator strength for compound $7 \mathrm{~b}$ in gas-phase and solvent medium.

\section{d) Computed molecular volume}

The molecular volume of each molecular structure was computed by using the VOLUME keyword on Gaussian 16 program package ${ }^{13}$. This calculation was requested after the optimization of the equilibrium geometries of each compound at the CAMB3LYP/6-311+G(d,p) level of theory. Also, the molecular volume wascalculated only in a solvent medium by employing the PCM.

\begin{tabular}{ccc}
\hline Compound & $\boldsymbol{a}^{\mathbf{3}}\left(\boldsymbol{A}^{\mathbf{3}}\right)$ experimental & $\boldsymbol{a}^{\mathbf{3}}\left(\boldsymbol{A}^{\mathbf{3}}\right)$ computed \\
\hline 3a in DCM & $250 \pm 50$ & 219.25 \\
\hline 3b in DCM & $270 \pm 60$ & 213.84 \\
\hline 3c in DCM & $280 \pm 90$ & 200.20 \\
\hline 3d in DCM & $220 \pm 40$ & 229.22 \\
\hline
\end{tabular}




\begin{tabular}{rcc}
\hline 3e in DCM & $250 \pm 60$ & 229.22 \\
\hline 3f in DCM & $320 \pm 100$ & 238.32 \\
\hline 3g in DCM & $220 \pm 60$ & 275.89 \\
\hline 7a in DMSO & $180 \pm 50$ & 195.11 \\
\hline 7b in DMSO & $190 \pm 60$ & 178.45 \\
\hline
\end{tabular}

Table S21: The molecular volume of each molecular structure.

\section{References}

(1) Zucolotto Cocca, L. H.; Ayhan, M. M.; Gürek, A. G.; Ahsen, V.; Bretonnière, Y.; de Paula Siqueira, J.; Gotardo, F.; Mendonça, C. R.; Hirel, C.; De Boni, L. Mechanism of the Zn(II)Phthalocyanines' Photochemical Reactions Depending on the Number of Substituents and Geometry. Molecules 2016, 21 (5), 635.

(2) de Souza, T. G. B.; Vivas, M. G.; Mendonça, C. R.; Plunkett, S.; Filatov, M. A.; Senge, M. O.; De Boni, L. Studying the Intersystem Crossing Rate and Triplet Quantum Yield of Meso-Substituted Porphyrins by Means of Pulse Train Fluorescence Technique. J. Porphyr. Phthalocyanines 2016, 20, 282-291.

(3) Abegão, L. M. G.; Fonseca, R. D.; Ramos, T. N.; Mahuteau-Betzer, F.; Piguel, S.; Joatan R., J.; Mendonça, C. R.; Canuto, S.; Silva, D. L.; De Boni, L. Oxazole Dyes with Potential for Photoluminescence Bioprobes: A Two-Photon Absorption Study. J. Phys. Chem. C 2018, 122 (19), 10526-10534.

(4) Cocca, L. H. Z.; Oliveira, T. M. A.; Gotardo, F.; Teles, A. V; Menegatti, R.; Siqueira, J. P.; Mendonça, C. R.; Bataus, L. A. M.; Ribeiro, A. O.; Souza, T. F. M.; Souza, G. R. L.; Gonçalves, P. J.; De Boni, L. Tetracarboxy-Phthalocyanines: From Excited State Dynamics to Photodynamic Inactivation against Bovine Herpesvirus Type 1. J. Photochem. Photobiol. B 2017, 175, 1-8.

(5) Dos, F.; Carlos, S.; Nunes, M. C.; De Boni, L.; Machado, G. S.; Souza Nunes, F. A Novel Fluorene-Derivative Schiff-Base Fluorescent Sensor for Copper (II) in Organic Media. J. Photochem. Photobiol. A 2017, 348, 41-46.

(6) Lakowicz, J. R.; Masters, B. R. Principles of Fluorescence Spectroscopy, Third Edition. J. Biomed. Opt. 2008, 13 (2), 029901.

(7) Islam, M. A. Einstein-Smoluchowski Diffusion Equation: A Discussion. Phys. Scr. 2004, 70 (2-3), 120-125.

(8) Vabre, R.; Legraverend, M.; Piguel, S. Synthesis and Evaluation of Spectroscopic Properties of Newly Synthesized Push-Pull 6-Amino-8-Styryl Purines. Dye. Pigment. 2014, 105 (105), 145-151.

(9) Molecular and Laser Spectroscopy; Elsevier, 2018.

(10) Neves, U. M.; De Boni, L.; Ye, Z.; Bu, X. R.; Mendonça, C. R. Two-Photon Absorption 
Spectra of Salen Dye Complexes with Azo Dyes. Chem. Phys. Lett. 2007, 441 (4-6), 221-225.

(11) Sheik-Bahae, M.; Said, A. A.; Wei, T.-H.; Hagan, D. J.; Van Stryland, E. W. Sensitive Measurement of Optical Nonlinearities Using a Single Beam. IEEE J. Quantum Electron. 1990, 26 (4), 760-769.

(12) Sheik-bahae, M.; Said, A. A.; Van Stryland, E. W. High-Sensitivity, Single-Beam N_2 Measurements. Opt. Lett. 1989, 14 (17), 955-957.

(13) M.J. Frisch, G.W. Trucks, H.B. Schlegel, G.E. Scuseria, M.A. Robb, J.R. Cheeseman, G. Scalmani, V. Barone, G.A. Petersson, H. N. Gaussian 16. Wallingford CT 2016. 